\title{
THE DEFORMED GRAPH LAPLACIAN AND ITS APPLICATIONS TO NETWORK CENTRALITY ANALYSIS
}

\author{
PETER GRINDROD*, DESMOND J. HIGHAM ${ }^{\dagger}$, AND VANNI NOFERINI ${ }^{\ddagger}$
}

\begin{abstract}
We introduce and study a new network centrality measure based on the concept of nonbacktracking walks; that is, walks not containing subsequences of the form $u v u$ where $u$ and $v$ are any distinct connected vertices of the underlying graph. We argue that this feature can yield more meaningful rankings than traditional walk-based centrality measures. We show that the resulting Katz-style centrality measure may be computed via the so-called deformed graph Laplacian-a quadratic matrix polynomial that can be associated with any graph. By proving a range of new results about this matrix polynomial, we gain insights into the behavior of the algorithm with respect to its Katz-like parameter. The results also inform implementation issues. In particular we show that, in an appropriate limit, the new measure coincides with the nonbacktracking version of eigenvector centrality introduced by Martin, Zhang and Newman in 2014. Rigorous analysis on star and star-like networks illustrates the benefits of the new approach, and further results are given on real networks.
\end{abstract}

Keywords: Centrality index, deformed graph Laplacian, matrix polynomial, nonbacktracking, complex network, generating function.

MSC classification: 05C50, 05C82, 15A18, 15A54, 15B48, 15B99, 65F15

1. Introduction. Network science is producing a wide range of challenging research problems that have diverse applications across science and engineering. Many important questions in this area can be cast in terms of applied linear algebra. In particular, operating on networks leads us naturally into the rich and elegant field of matrix function theory. In this work, we consider network centrality measures, a topic where many tools have been developed and tested [19, 47]. We focus on walkbased centrality measures $[12,20,22]$. Here the aim is to identify influential nodes by quantifying their potential to disperse information along the network edges. A key novelty in our work is to ignore certain types of walk around the network that, in terms of quantifying centrality, have little relevance. The combinatorics of the remaining "nonbacktracking walks" can be dealt with conveniently via a matrix polynomial representation, leading to an efficient computational algorithm. This viewpoint also allows us to raise and solve several new theoretical problems on this matrix polynomial, giving further insight into the algorithm.

In section 2, we review some relevant material on walk-based centrality measures, focusing on Katz and eigenvector centrality. In section 3, we then motivate a new definition of centrality based on the concept of a nonbacktracking walk. We show on a simple star graph how restricting attention to nonbacktracking walks can avoid a localization issue. Section 4 provides some preliminary material on matrix polynomials and, in particular, sets up the so-called deformed graph Laplacian, which is the main object of study in our work, and derives some new basic connections between the eigenvalues of the deformed graph Laplacian and the features of the underlying graph. In section 5, we consider the combinatorics of nonbacktracking walks, and show how an analogue of Katz centrality can be expressed in terms of the deformed graph Laplacian.

*Mathematical Institute, University of Oxford, Andrew Wiles Building, Radcliffe Observatory Quarter, Woodstock Road, Oxford, UK, OX2 6GG. (grindrod@maths.ac.uk)

${ }^{\dagger}$ Department of Mathematics and Statistics, University of Strathclyde, Glasgow, UK, G1 1XH. (d.j.higham@strath.ac.uk) Supported by EPSRC/RCUK Established Career Fellowship EP/M00158X/1 and by a Royal Society/Wolfson Research Merit Award.

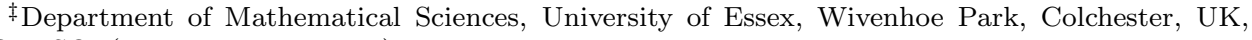
CO4 3SQ. (vnofer@essex.ac.uk) 
Relevant properties of the deformed graph Laplacian are then studied in sections 6, 7, 8, 9 and 10. More specifically, sections 6 and 7 contain our main theoretical results, exploring further the spectral properties of the deformed graph Laplacian, and connecting them to the radius of convergence of a certain power series that generates the combinatorics of nonbacktracking walks. In section 8 we consider how to compute or bound the radius of convergence, and discuss other practical issues. Section 9 develops the relation between the deformed graph Laplacian and M-matrix theory. We show in section 10 that the nonbacktracking version of eigenvector centrality introduced by Martin, Zhang and Newman [33], which was derived from a different viewpoint, coincides with the nonbacktracking walk centrality in an appropriate limit. A large scale synthetic example is then analysed in section 11, in order to shed further light on the new centrality measure, and tests on real data are given in section 12 . We finish in section 13 with a short summary.

It is worth mentioning that Theorem 6.1 and slightly weaker versions of Theorem 4.7 and Lemma 6.2 are not entirely new. They can also be obtained using purely graph theoretical techniques based on zeta funtions, namely, Theorem 2 in [43]. We opted, however, to include our own proofs based on matrix theory, as they allow us to prove stronger statements and present a self-contained treatment. We also emphasize that the other theoretical results in this paper are, to our knowledge, new.

2. Walk-based Centrality. We let $A \in \mathbb{R}^{n \times n}$ denote the adjacency matrix of a simple, undirected graph, so $a_{i j}=1$ if there is an edge from node $i$ to node $j$ and $a_{i j}=0$ otherwise. A walk of length $k$ is any sequence of $k+1$ nodes, $i_{1}, i_{2}, \ldots, i_{k+1}$ such that each edge $i_{r} \leftrightarrow i_{r+1}$ is present in the network [11, 14, 19]. Loosely, a walk is a traversal around the network in which nodes and edges may be re-used.

We may, of course, place further restrictions on the traversal - a trail must use distinct edges, a path must use distinct nodes and a shortest path must use the smallest possible number of edges. Borgatti [11] discusses the relevance of these concepts with respect to processes that take place over a network, such as message-passing, diseasespreading and various types of business transaction. This variety of processes has led to a wide range of centrality measures that aim to summarize the importance of the network nodes through their ability to initiate traversals. We focus here on the case of walks for two main reasons. First, walks are relevant in many realistic circumstances, notably, where there is a stochastic element to the dynamics; for example, a fixed object such as a soccer ball, an office laptop or the keys to a company car may be passed arbitrarily around a well-defined interaction network. Second, and from a more practical perspective, walks are convenient to compute with, making it feasible to study the type of large-scale networks arising in modern applications.

A classic result from graph theory tells us that $\left(A^{k}\right)_{i j}$ counts the number of distinct walks of length $k$ from $i$ to $j$; see, for example, [14, Theorem 2.2.1]. Now, let $\rho(A)$ denote the spectral radius of $A$ and suppose $0<\alpha<\rho(A)^{-1}$. Then the resolvent

$$
(I-\alpha A)^{-1}=I+\alpha A+\alpha^{2} A^{2}+\alpha^{3} A^{3}+\cdots
$$

has an $i, j$ element that records a weighted sum of all walks ${ }^{1}$ from $i$ to $j$, with walks of length $k$ downweighted by the factor $\alpha^{k}$. Katz [28] suggested that the importance, or centrality, of node $i$ could be quantified by summing this count over all such $j$,

\footnotetext{
${ }^{1}$ For convenience, we have included an identity matrix term in the expansion. This may be regarded as representing a single closed walk of length zero.
} 
leading to the linear system

$$
(I-\alpha A) \mathbf{x}=\mathbf{1}
$$

Here, $\mathbf{1}$ is the vector whose components are all 1 while $x_{i}>0$ denotes the centrality of node $i$, and the relative size of the components in $x$ can be used for ranking. Katz [28] also pointed out that the attenuation parameter $\alpha$ may be interpreted as the independent probability that an edge is traversed effectively; so the probability of a walk of length $k$ succeeding will be $\alpha^{k}$.

Under the assumption that the network is connected, letting the attenuation parameter $\alpha$ approach $1 / \rho(A)$ from below in (2.2) we arrive at the eigenvector centrality measure, introduced by Bonacich [9, 10, 47], where $\mathbf{x}$ matches the Perron-Frobenius eigenvector of $A$. So $A \mathbf{x}=\lambda \mathbf{x}$, where $\lambda=\rho(A)$ and $x_{i}>0$.

3. Nonbacktracking Walks. The sum (2.1) includes some traversals that, intuitively, are less relevant than others. In particular, for every edge $i \leftrightarrow j,(2.1)$ incorporates all walks that pass from $i$ to $j$ and immediately pass back to $i$, rather than exploring other parts of the network. We argue that, from the perspective of walk-counting centrality, such traversals are best ignored, leading to Definitions 3.1 and 3.2 below. We note that similar arguments, albeit from a spectral graph theory perspective rather than from the point of view of combinatoric walk-counting, were given in [33], where a nonbactracking version of eigenvector centrality was proposed. We explore further the connection between our work and [33] in section 10.

In a different setting, [1] considers nonbacktracking random walks around a regular graph, whereas our work concerns the combinatorics of (deterministic) traversals as a means to quantify centrality. As we mention in section 5, nonbactracking walks have also been studied in the theory of zeta functions of graphs [26, 43].

DEFINITION 3.1. A backtracking walk is a walk that contains at least one node subsequence of the form uvu, i.e., it visits $u, v$ and then $u$ in immediate succession.

A nonbacktracking walk is a walk that is not backtracking, i.e., it does not contain any subsequence of the form uvu.

For brevity we will henceforth replace the phrase nonbacktracking walk with NBTW.

DEFINITION 3.2. For an appropriate value of the real parameter $t>0$, the NBTW centrality of node $i$ is defined by

$$
1+\sum_{j=1}^{n} \sum_{k=1}^{\infty} t^{k}\left(p_{k}(A)\right)_{i j}
$$

where $\left(p_{k}(A)\right)_{i j}$ records the number of distinct NBTWs of length $k$ from $i$ to $j$.

In subsequent sections we will show how to compute NBTW centrality in terms of a certain matrix polynomial depending on the original adjacency matrix, $A$, and study the role of the parameter $t$. At this stage, we simply note that $0<t<1$ is a natural requirement, so that longer walks carry less weight, and we continue with an illustrative example that differentiates the new measure from Katz centrality.

Consider a star graph with $n$ nodes, as illustrated for the case $n=9$ in Figure 3.1. Here the central hub node has an undirected edge to each of the $n-1$ leaf nodes. The 
adjacency matrix has the form

$$
A=\left[\begin{array}{cccc} 
& 1 & \cdots & 1 \\
1 & & & \\
\vdots & & & \\
1 & & &
\end{array}\right] \in \mathbb{R}^{n \times n}
$$

where a blank denotes a zero entry. The eigenvalues of $A$ are $\pm \sqrt{n-1}$ and 0 (repeated $n-2$ times); see section 11 for a more general case. Hence, Katz centrality (2.2) is defined for $0<\alpha<1 / \sqrt{n-1}$.

By symmetry the $x_{i}$ values in the Katz system (2.2) are equal for all $i \geq 2$, and the equations reduce to $x_{1}-\alpha(n-1) x_{2}=1$ and $x_{2}-\alpha x_{1}=1$. These solve to give

$$
x_{1}=\frac{1+\alpha(n-1)}{1-\alpha^{2}(n-1)} \quad \text { and } \quad x_{i}=\frac{1+\alpha}{1-\alpha^{2}(n-1)}, \quad \text { for } i \geq 2 .
$$

The ratio of hub centrality to leaf centrality is therefore, for $i \geq 2$,

$$
\frac{x_{1}}{x_{i}}=\frac{1+\alpha(n-1)}{1+\alpha} \text {. }
$$

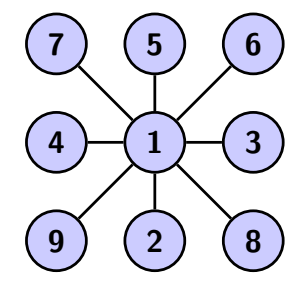

FIG. 3.1. A star graph with $n=9$ vertices.

Turning to NBTWs, for the star graph it follows directly from Definition 3.1 that

- node 1 has $n-1$ NBTWs of length one and no NBTWs of length greater than one,

- node $i$ for $i \geq 2$ has one NBTW of length one, $n-2$ NBTWs of length two, and no NBTWs of length greater than two.

Hence, in Definition 3.2 the NBTW centralities are

$$
x_{1}=1+(n-1) t \quad \text { and } \quad x_{i}=1+t+(n-2) t^{2}, \quad \text { for } i \geq 2 .
$$

So the ratio of hub centrality to leaf centrality is, for $i \geq 2$,

$$
\frac{x_{1}}{x_{i}}=\frac{1+(n-1) t}{1+t+(n-2) t^{2}} \text {. }
$$

We are interested in large systems, so consider the limit $n \rightarrow \infty$. In the Katz regime we require $\alpha<1 / \sqrt{n-1}$. If we take $\alpha$ to be a fixed proportion of this upper limit, say $0.9 / \sqrt{n-1}$, then in (3.2) and (3.3) we have

$$
x_{1}=O(\sqrt{n}), \quad x_{i}=O(1), \quad x_{1} / x_{i}=O(\sqrt{n}) .
$$

Similarly, for the NBTW version, using $t=0.9 / \sqrt{n-1}$ in (3.4) and (3.5) we obtain the same asymptotic behaviour. However, in this example the NBTW centrality 
measure is valid for any $t$. So we may consider the case where $t=O(1)$ as $n \rightarrow \infty$, e.g., $t=1 / 2$, in which case

$$
x_{1}=O(n), \quad x_{i}=O(n), \quad x_{1} / x_{i} \rightarrow \frac{1}{t}=O(1) .
$$

So, compared with Katz, the NBTW measure

- has a much less severe restriction on the downweighting parameter, and

- for fixed $t$ and large $n$, gives a less dramatic distinction between the hub and the leaves.

For a vector $v \in \mathbb{R}^{n}$ with $v_{i} \geq 0$ and $\|v\|_{2}=1$, the inverse participation ratio, defined as

$$
S=\sum_{i=1}^{n} v_{i}^{4},
$$

was used in [33] to quantify the phenomenon of localization, where most of the weight is concentrated on a small number of components (in our example, a single network node). The Katz centrality vector in (3.6), exhibits localization, in the sense of [33], since $S=O(1)$, whereas the NBTW centrality vector (3.7), with $S=O(1 / n)$, does not. The authors in [33] put forward the view that in the context of centrality measures localization is "undesirable, significantly diminishing the effectiveness of the centrality as a tool for quantifying the importance of the nodes." In terms of using a centrality measure to rank nodes - for example, picking out a small number of big hitters, or comparing two nodes that are of particular interest - it may be argued that localization of measure is not in itself a drawback if the relative values are meaningful, allowing us to distinguish between components. Indeed, for the star graph, both measures always rate the hub node most highly. In section 11, however, we give a more general example where Katz and NBTW centrality can produce different rankings, showing that the two measures are distinct in a more fundamental sense.

4. Matrix Polynomials and the Deformed Graph Laplacian. We now provide some general background material on matrix polynomials before introducing and studying the deformed graph Laplacian. Recall that, given a field $\mathbb{F}$ (in this paper, $\mathbb{F}$ is either $\mathbb{R}$ or $\mathbb{C}$ ), the set of univariate polynomials in $t$ with coefficients in $\mathbb{F}$ is denoted by $\mathbb{F}[t]$. Moreover, the set of square matrices of size $n$ with entries in $\mathbb{F}[t]$ is denoted by $\mathbb{F}[t]^{n \times n}$.

For $j=0,1, \ldots, k$, let $A_{j} \in \mathbb{C}^{n \times n}$ be square matrices of the same size with $A_{k} \neq 0$. The matrix-valued function $P(t)=\sum_{j=0}^{k} A_{j} t^{j} \in \mathbb{C}[t]^{n \times n}$ is called a square matrix polynomial of degree $k$. If $\operatorname{det} P(t) \equiv 0$ then $P(t)$ is said to be singular, otherwise it is called regular. We now recall some basic definitions from the spectral theory of regular matrix polynomials [24].

The finite eigenvalues of a square regular matrix polynomial of degree $k$ are the zeros of the scalar polynomial $\operatorname{det} P(t)$. Moreover, if $\operatorname{deg} \operatorname{det} P(t)<k n$, we say that $\infty$ is an eigenvalue of $P(t)$. If a finite eigenvalue has multiplicity 1 as a root of $\operatorname{det} P(t)$ it is called a simple eigenvalue; similarly, an infinite eigenvalue is simple if $\operatorname{deg} \operatorname{det} P(t)=k n-1$. An eigenvalue which is not simple is called multiple. If $\lambda \in \mathbb{C}$ is a finite eigenvalue of $P(t)$, any nonzero vector $\mathbf{v} \in \mathbb{C}^{n}$ such that $P(\lambda) \mathbf{v}=\mathbf{0}$ is called an eigenvector associated with the eigenvalue $\lambda$. Similarly, if $P(t)$ has at least one infinite eigenvalue, then any nonzero vector $\mathbf{w} \in \mathbb{C}^{n}$ such that $A_{k} \mathbf{w}=\mathbf{0}$ is an eigenvector associated with the eigenvalue $\infty$. 
The algebraic multiplicity of a finite (resp., infinite) eigenvalue of $P(t)$ is the multiplicity of the eigenvalue as a root of $\operatorname{det} P(t)$ (resp., the number $k n-\operatorname{deg} \operatorname{det} P(t)$ ). Moreover, we say that a finite eigenvalue $\lambda \in \mathbb{C}$ has geometric multiplicity $n-$ $\operatorname{rank} P(\lambda)$, and similarly the eigenvalue infinity has geometric multiplicity $n-\operatorname{rank} A_{k}$. An eigenvalue has geometric multiplicity $g$ if and only if one can find $g$ linearly independent eigenvectors associated with it. If the algebraic and geometric multiplicities of an eigenvalue coincide, we say that the eigenvalue is semisimple; otherwise, it is defective.

It is easy to check that, by the definitions above, a regular matrix polynomial of size $n$ and degree $k$ has precisely $k n$ eigenvalues, counted with their algebraic multiplicities and possibly including infinite eigenvalues. In this paper, we will focus on a real matrix polynomial, i.e., $A_{i} \in \mathbb{R}^{n \times n}$. Note that, even if $P(t) \in \mathbb{R}[t]^{n \times n}$, the variable $t \in \mathbb{C}$ is generally allowed to be complex, and a real matrix polynomial may have nonreal finite eigenvalues.

More details on the spectral theory of matrix polynomials can be found in, e.g., $[24,32,37,38]$ and the references therein. The following result, which is a special case of the Smith Canonical Form Theorem [23], will be needed below.

THEOREM 4.1. Let $P(t) \in \mathbb{R}[t]^{n \times n}$ be an arbitrary real regular matrix polynomial. Then, there exist two unimodular, i.e., with constant nonzero determinant, real matrix polynomials $E(t)$ and $F(t)$ of size $n \times n$ such that

$$
E(t) P(t) F(t)=S(t):=\operatorname{diag}\left(\ell_{1}(t), \ell_{2}(t), \ldots, \ell_{n}(t)\right),
$$

where $\ell_{i}(t) \in \mathbb{R}[t]$, called the invariant polynomials of $P(t)$, are monic polynomials with the property that $\ell_{i}(t)$ is a divisor of $\ell_{i+1}(t)$ for all $i=1, \ldots, n-1$. Moreover, letting $g_{0}(t)=1$ and, for $i=1, \ldots, n$, letting $g_{i}(t)$ denote the monic greatest common divisor of all the minors of $P(t)$ of order $i$, the invariant polynomials are given by the formulae $\ell_{i}(t)=g_{i}(t) / g_{i-1}(t)$.

The next property of the last invariant polynomial will play a role in what follows.

Proposition 4.2. Let $P(t) \in \mathbb{R}[t]^{n \times n}$ be a regular matrix polynomial with invariant polynomials $\ell_{1}(t), \ldots, \ell_{n}(t)$. Then, $\lambda \in \mathbb{C}$ is a finite eigenvalue of $P(t)$ if and only if $\ell_{n}(\lambda)=0$. Moreover, it is a semisimple finite eigenvalue of $P(t)$ if and only if it is a simple zero of $\ell_{n}(t)$.

Proof. Suppose $\ell_{n}(\lambda)=0$. It is clear by Theorem 4.1 that there exists a nonzero constant $\kappa \in \mathbb{R}$ such that $\operatorname{det} P(t)=\kappa \prod_{i=1}^{n} \ell_{i}(t)$, and hence $\operatorname{det} P(\lambda)=0$. Conversely, suppose that $\operatorname{det} P(\lambda)=0$. By the same argument there exists $j \in\{1,2, \ldots, n\}$ such that $\ell_{j}(\lambda)=0$. But since any invariant polynomial is a divisor of its successor, in particular $\ell_{j}(t)$ divides $\ell_{n}(t)$ for any $j$, and hence, $\ell_{n}(\lambda)=0$.

Finally, let $\alpha_{i}$ be the multiplicity of $\lambda$ as a zero of $\ell_{i}(t)$ for $i=1,2, \ldots, n$. Clearly, $\alpha_{1} \leq \alpha_{2} \leq \cdots \leq \alpha_{n}$. Moreover, the geometric multiplicity of $\lambda$ as an eigenvalue of $M(t)$ is $\sum_{i: \alpha_{i}>0} 1$ while its algebraic multiplicity is $\sum_{i=1}^{n} \alpha_{i}$. It follows that $\lambda$ is semisimple if and only if $\alpha_{i} \leq 1$ for all $i$, which is equivalent to $\alpha_{n}=1$.

If a square matrix polynomial $P(t)$ is such that $P(t)=P(t)^{*}$ for all $t \in \mathbb{R}$ then $P(t)$ is called Hermitian [24,34]. The spectral theory of Hermitian matrix polynomials is richer and subtler than the general case: see [24,34] and the references therein. We note here that, unlike for Hermitian matrices, the eigenvalues of Hermitian matrix polynomials are not necessarily all real. However, they do appear in complex conjugate pairs $[24,34]$.

Given an adjacency matrix $A$ of an undirected graph, here and below we denote by $\Delta$ the associated diagonal degree matrix; that is, $\Delta_{i i}:=\left(A^{2}\right)_{i i}$. The matrix 
$L:=\Delta-A$ is called the graph Laplacian; it is symmetric positive semidefinite, and its properties are well understood $[8,35]$. We now turn our attention to the deformed graph Laplacian: a special matrix polynomial associated with any graph. The deformed graph Laplacian has been studied in [36] because of its applications to consensus algorithms in multi-agent systems and robotics. Here, we will analyze it more thoroughly using the spectral theory of matrix polynomials, and we will then focus on its connections to NBTW centrality.

Definition 4.3. [36] Let $A \in \mathbb{R}^{n \times n}$ be the adjacency matrix of an undirected graph. For any $t \in \mathbb{C}$, the associated deformed graph Laplacian is the Hermitian matrix polynomial

$$
M(t)=I-A t+(\Delta-I) t^{2} \in \mathbb{R}[t]^{n \times n} .
$$

Observe that $M(1)=L$ is the graph Laplacian, $M(0)=I$ is the identity matrix, while $M(-1)$ is the signless graph Laplacian [15].

Proposition 4.4 records some basic spectral properties of $M(t)$ which were, in part, discussed also in [36].

PROPOSITION 4.4. The following hold:

1. $M(t)$ is a regular matrix polynomial, and 0 is never an eigenvalue of $M(t)$;

2. 1 is always an eigenvalue of $M(t)$, with geometric multiplicity equal to the number of connected components of the graph of $A$;

3. the geometric multiplicity of $\infty$ as an eigenvalue of $M(t)$ is equal to the number of leaves, i.e., vertices of degree 1, in the graph of $A$ (in particular, $\infty$ is an eigenvalue of $M(t)$ if and only if the graph of $A$ has at least one leaf);

4. -1 is an eigenvalue of $M(t)$ if and only if the graph of $A$ has at least one bipartite component. In this case, the geometric multiplicity of -1 is equal to the number of bipartite components of the graph of $A$.

Proof.

1. We have $\operatorname{det} M(0)=1$, and therefore $\operatorname{det}(M(t))$ cannot be the zero polynomial; moreover, by the same argument, 0 is not an eigenvalue.

2. Observe that the graph Laplacian $L$ is always a singular matrix, because $A \mathbf{1}=\Delta \mathbf{1}$. Therefore, $\operatorname{det} M(1)=\operatorname{det} L=0$. The nullity of $L$, and hence the geometric multiplicity of 1 as an eigenvalue of $M(t)$, is equal to the number of connected components in the graph of $A$ [35].

3. The geometric multiplicity of the infinite eigenvalue is the nullity of $\Delta-I$, which is equal to the number of leaves in the graph of $A$.

4. $M(t)$ has the eigenvalue $-1 \Leftrightarrow$ the signless graph Laplacian is a singular matrix $\Leftrightarrow$ the graph of $A$ has at least one bipartite component [15, Proposition $2.1]$; moreover the multiplicity of the eigenvalue 0 of the signless graph Laplacian, and hence the geometric multiplicity of -1 as an eigenvalue of $M(t)$, is equal to the number of bipartite components in the graph of A [15, Corollary $2.2]$.

In what follows, both in this section and in sections 6 and 7 , we will explore further the spectral properties of $M(t)$, obtaining several results that are, to our knowledge, new. The next proposition shows that, for disconnected graphs, it suffices to study the individual deformed graph Laplacians associated with each connected component.

Proposition 4.5. Let $A \in \mathbb{R}^{n \times n}$ be the adjacency matrix of a disconnected graph having $c$ connected components with adjacency matrices $A_{i}$, for $i=1, \ldots, c$; let 
$M(t)$ be the deformed graph Laplacian associated with $A$ and $M_{i}(t)$ be the deformed graph Laplacians associated with $A_{i}$, for $i=1, \ldots, c$; and let $\lambda \in \mathbb{C} \cup\{\infty\}$. Then, $\lambda$ is an eigenvalue of $M(t)$ if and only if it is an eigenvalue of $M_{i}(t)$ for some value of $i=1, \ldots, c$. Moreover, denote by $\gamma(\lambda)$ (resp. $\gamma_{i}(\lambda)$ ) the geometric multiplicity of $\lambda$ as an eigenvalue of $M(t)$ (resp. $M_{i}(t)$ ). Similarly, let $\alpha(\lambda)$ and $\alpha_{i}(\lambda)$ denote the corresponding algebraic multiplicities. Then, it holds

$$
\gamma(\lambda)=\sum_{i=1}^{c} \gamma_{i}(\lambda), \quad \alpha(\lambda)=\sum_{i=1}^{c} \alpha_{i}(\lambda)
$$

Proof. Since the underlying graph is disconnected, by relabelling the nodes we see that $A$ (resp., $M(t)$ ) is permutation similar to a block diagonal matrix (resp., matrix polynomial) whose diagonal blocks have sizes equal to the sizes of each connected component of the underlying graph. Clearly, each diagonal block $M_{i}(t)$ is precisely the deformed graph Laplacian associated with the $i$ th connected component. If $\lambda \in$ $\mathbb{C}$, the statement is an immediate consequence of the observation above and of the definitions of multiplicities of an eigenvalue. If $\lambda=\infty$, the statement about the algebraic multiplicity requires a little extra care. To see it, note that denoting by $n_{i}$ the size of the $i$ th connected component of the graph

$$
\alpha(\infty)=2 n-\operatorname{deg} \operatorname{det} M(t)=2\left(\sum_{i=1}^{c} n_{i}\right)-\left(\sum_{i=1}^{c} \operatorname{deg} \operatorname{det} M_{i}(t)\right)=\sum_{i=1}^{c} \alpha_{i}(\infty) .
$$

Proposition 4.4 does not say anything about the algebraic multiplicities of the special eigenvalues $1,-1, \infty$. We complete the picture with Proposition 4.6, which characterizes when these special eigenvalues are semisimple or defective in terms of features of the underlying graph.

Proposition 4.6.

1. The eigenvalue 1 of $M(t)$ is semisimple if and only if there is no connected component of the graph of $A$ having average degree precisely equal to 2 ; and it is simple if and only if the previous condition holds and the graph of $A$ is connected.

2. Suppose that -1 is an eigenvalue of $M(t)$. Then, it is semisimple if and only if there is no bipartite connected component of the graph of A having average degree precisely equal to 2 ; and it is simple if and only if the previous condition holds and the graph of $A$ has only one bipartite connected component.

3. Suppose that $\infty$ is an eigenvalue of $M(t)$. Then, it is semisimple if and only if every connected component of the graph of A which has a leaf is the complete graph with 2 vertices. Moreover, $\infty$ can never be a simple eigenvalue.

Proof. By Proposition 4.5 we may assume without loss of generality that the graph of $A$ is connected.

Our proof is based on the following classical results in the theory of matrix polynomials [24]: a finite eigenvalue $\lambda$ of the matrix polynomial $P(t)=\sum_{i=1}^{k} A_{t} t^{i}$, associated with the eigenvector $\mathbf{w}$, is defective if and only if there exists ${ }^{2}$ a vector $\mathbf{v}$ such that

$$
P(\lambda) \mathbf{v}+P^{\prime}(\lambda) \mathbf{w}=\mathbf{0}
$$

\footnotetext{
${ }^{2}$ Unlike for Jordan chains of matrices, here there is no linear independence condition, and in fact, the Jordan vector $\mathbf{v}$ may also be the zero vector, see [24].
} 
Similarly, the eigenvalue $\infty$, associated with the eigenvector $\mathbf{w}$, is defective if and only if there exists a vector $\mathbf{v}$ such that

$$
A_{k} \mathbf{v}+A_{k-1} \mathbf{w}=\mathbf{0} .
$$

Hence:

1. The eigenvalue 1 of $M(t)$ is associated with the eigenvector $\mathbf{1}$. Noting that $M(1)=L$ and $M^{\prime}(1)=2 L+A-2 I,(4.2)$ becomes $L \mathbf{v}+A \mathbf{1}=2 \cdot \mathbf{1}$. The latter equation has a solution if and only if $(A-2 I) \mathbf{1}$ lies in the column space of $L$, or equivalently, $0=\mathbf{1}^{T}(A-2 I) \mathbf{1}=d-2 n$, where $d=\sum_{i=1}^{n} \operatorname{deg}_{i}$ is the sum of the degrees of all the nodes. Hence, 1 is defective if and only if $d / n=2$, i.e., the average degree is 2 .

2. The proof is analogous to the previous case, noting that if -1 is an eigenvalue of $M(t)$ associated with a connected graph, then, since $M(-1)=: Q$ is the signless graph Laplacian, the graph is bipartite and the associated eigenvector $\mathbf{w}$ is such that $w_{i}=-w_{j}$ for every edge $i \leftrightarrow j[15]$. Since $M^{\prime}(-1)=A-2 Q+$ $2 I$, we see that -1 is defective if and only if $0=\mathbf{w}^{T}(A+2 I) \mathbf{w}=-d+2 n$.

3. This time, we start from (4.3) and note that $A_{k}=\Delta-I$ and $A_{k-1}=-A$. Clearly, for every node $i$ which is a leaf $\mathbf{e}_{i}$ is an eigenvector associated with $\infty$. Then, $\infty$ is defective $\Leftrightarrow$ the equation $(\Delta-I) \mathbf{v}=A \mathbf{e}_{i}$ has a solution $\Leftrightarrow$ $A \mathbf{e}_{i}$ is orthogonal to $\mathbf{e}_{j}$ for every $j$ which is in turn a leaf $\Leftrightarrow$ the unique node connected to $i$ is not itself a leaf. The only way for a connected graph to have two leaves connected to each other (and hence for $\infty$ to be a semisimple eigenvalue) is if the graph is the complete graph with two vertices. In this case, however, $\infty$ must have both algebraic and geometric multiplicity 2 .

We now give a powerful auxiliary result.

THEOREM 4.7. Let $A$ be the adjacency matrix of a simple, undirected, connected graph. Denote by $\widetilde{A}$ the adjacency matrix, possibly of smaller size, such that the graph of $\widetilde{A}$ is obtained by removing from the graph of $A$ all the leaves, if any, and the edges connecting these leaves to the rest of the graph. Suppose that the graph of $\widetilde{A}$ is not empty, i.e., it contains at least one node. Let $M(t), \widetilde{M}(t)$ be the deformed graph Laplacians associated with $A, \widetilde{A}$ respectively.

Then, $\lambda \in \mathbb{C}$ is a finite eigenvalue of $M(t)$ if and only if it is a finite eigenvalue of $\widetilde{M}(t)$. Moreover, the geometric multiplicities of $\lambda$ as an eigenvalue of $M(t)$ and $\widetilde{M}(t)$ are the same.

Proof. Suppose that there are $\ell$ leaves in the graph of $A$. If $\ell=0$, there is nothing to prove. Furthermore, the graph of $\widetilde{A}$ is not empty unless $\ell=n$ (and $n=2$ ). Hence, we may assume $0<\ell<n$. Moreover, without loss of generality, we label the leaves in the graph of $A$ as nodes $1, \ldots, \ell$.

Now, $\lambda \in \mathbb{C}$ is a finite eigenvalue of $M(t)$ if and only if there exists a nonzero $\mathbf{v} \in \mathbb{C}^{n}$ such that

$$
\lambda^{2}(\Delta-I) \mathbf{v}-A \lambda \mathbf{v}+\mathbf{v}=\mathbf{0} .
$$

Let $\operatorname{deg}_{i}$ denote the degree of node $i$. Clearly, $\operatorname{deg}_{i}=1$ for $i \leq \ell$, while for $i>\ell$ we set $\operatorname{deg}_{i}=\ell_{i}+\nu_{i}$ where $\ell_{i}$ is the number of leaves adjacent to node $i$. Hence, (4.4) is equivalent to the following scalar equations: for $i \leq \ell, v_{i}=\lambda v_{j}$, where $j$ is the unique node adjacent to node $i$, while for $i>\ell$

$$
0=\left(\ell_{i}+\nu_{i}-1\right) \lambda^{2} v_{i}+v_{i}-\lambda \sum_{j} v_{j}=\left(\ell_{i}+\nu_{i}-1\right) \lambda^{2} v_{i}-\lambda^{2} \ell_{i} v_{i}+v_{i}-\lambda \sum_{k} v_{k},
$$


where the first summation is over all $j$ such that node $j$ is adjacent to node $i$, while the second summation is over all $k$ such that node $k$ is not a leaf and is adjacent to node $i$. We rewrite the equations associated with $i>\ell$ as

$$
\left(\nu_{i}-1\right) \lambda^{2} v_{i}+v_{i}-\lambda \sum_{k} v_{k}
$$

to see that a nonzero solution to (4.4) exists if and only if there is a nonzero $\widetilde{\mathbf{v}} \in \mathbb{C}^{n-\ell}$ such that

$$
\lambda^{2}\left(\widetilde{\Delta}-I_{n-\ell}\right) \widetilde{\mathbf{v}}-\lambda \widetilde{A} \widetilde{\mathbf{v}}+\widetilde{\mathbf{v}}=\mathbf{0},
$$

where $\widetilde{\Delta}=\operatorname{diag}\left(\operatorname{diag}\left(\widetilde{A}^{2}\right)\right)$. To conclude the proof of the first statement, we note that the latter holds if and only if $\lambda$ is an eigenvalue of $\widetilde{M}(t)$.

By replicating the argument above $\gamma$ times, we now build two sets of $\gamma$ nonzero vectors each, say, $\mathbf{v}_{(1)}, \mathbf{v}_{(2)}, \ldots \mathbf{v}_{(\gamma)} \in \mathbb{C}^{n}$, and $\widetilde{\mathbf{v}}_{(1)}, \widetilde{\mathbf{v}}_{(2)}, \ldots \widetilde{\mathbf{v}}_{(\gamma)} \in \mathbb{C}^{n-\ell}$, such that for $j=1, \ldots, \gamma$,

$$
M(\lambda) \mathbf{v}_{(j)}=\mathbf{0}, \quad \widetilde{M}(\lambda) \widetilde{\mathbf{v}}_{(j)}=\mathbf{0}, \quad \mathbf{v}_{(j)}=\left[\begin{array}{c}
\star \\
\widetilde{\mathbf{v}}_{(j)}
\end{array}\right]
$$

where here and below $\star$ denotes a block whose exact form is not relevant. The first set of vectors is linearly independent if and only if the second is. Indeed,

$$
\begin{gathered}
{\left[\begin{array}{llll}
\mathbf{v}_{(1)} & \mathbf{v}_{(2)} & \ldots & \mathbf{v}_{(\gamma)}
\end{array}\right]=\left[\begin{array}{c}
\star \\
I_{n-\ell}
\end{array}\right]\left[\begin{array}{llll}
\widetilde{\mathbf{v}}_{(1)} & \widetilde{\mathbf{v}}_{(2)} & \ldots & \widetilde{\mathbf{v}}_{(\gamma)}
\end{array}\right]} \\
\Rightarrow \operatorname{rank}\left[\begin{array}{llll}
\mathbf{v}_{(1)} & \mathbf{v}_{(2)} & \ldots & \mathbf{v}_{(\gamma)}
\end{array}\right]=\operatorname{rank}\left[\begin{array}{llll}
\widetilde{\mathbf{v}}_{(1)} & \widetilde{\mathbf{v}}_{(2)} & \ldots & \widetilde{\mathbf{v}}_{(\gamma)}
\end{array}\right]
\end{gathered}
$$

This proves the statement on the the geometric multiplicities. $\square$

Theorem 4.7 says that to compute the finite eigenvalues of $M(t)$ we are allowed to remove all the leaves of the underlying graph, and iterate the process until we are left with a graph with no leaves. (As a consequence, if the underlying graph is a forest, then the only finite eigenvalues are \pm 1 , which must be both semisimple. This observation is recorded as Corollary 7.1 in section 7, with an alternative proof based on the connection with NBTWs.) As we discuss in section 8, the fact that the spectrum of the matrix polynomial does not "see" the leaves in a graph also has useful practical implications.

As our first application of Theorem 4.7, we show that the deformed graph Laplacian can never have finite eigenvalues of modulus larger than 1.

THEOREM 4.8. Let $M(t)$ be the deformed graph Laplacian associated with a simple undirected graph. Suppose that $\lambda \in \mathbb{C}$ is a finite eigenvalue of $M(t)$. Then, $|\lambda| \leq 1$.

Proof. By Proposition 4.5 and Theorem 4.7, we may assume with no loss of generality that the graph of $A$ is connected and that it does not have any leaves.

If $\lambda \in \mathbb{C}$ is a finite eigenvalue of $M(t)$, then there exists a nonzero $\mathbf{v} \in \mathbb{C}^{n}$ such that (4.4) holds. Without loss of generality we take $\|\mathbf{v}\|_{2}=1$. Premultiplying (4.4) by $\mathbf{v}^{*}$, we obtain

$$
\alpha \lambda^{2}-\beta \lambda+1=0
$$


where $\alpha=\mathbf{v}^{*} \Delta \mathbf{v}-1$ and $\beta=\mathbf{v}^{*} A \mathbf{v}$. Denoting the degree of the $i$ th node by $\operatorname{deg}_{i}$, we have $\operatorname{deg}_{i} \geq 2$ for all $i$, and hence $\alpha=\sum_{i=1}^{n} \operatorname{deg}_{i}\left|v_{i}\right|^{2}-1 \geq 2-1=1$.

There are two cases. If $\lambda \notin \mathbb{R}$, then $\lambda^{*}$ is also an eigenvalue of $M(t)$ and a root of (4.5). It follows that $1 \leq \alpha=|\lambda|^{-2} \Leftrightarrow|\lambda| \leq 1$. Suppose now $\lambda \in \mathbb{R}$. Using also the fact that $\Delta \pm A$ are both positive semidefinite matrices [15, 35], which implies $|\beta| \leq \alpha+1$, we have $0 \leq \beta^{2}-4 \alpha \leq(\alpha-1)^{2}$, and hence, $|\lambda| \leq\left(|\beta|+\sqrt{\beta^{2}-4 \alpha}\right) /(2 \alpha) \leq$ $(\alpha+1+\alpha-1) /(2 \alpha)=1$.

5. Nonbacktracking Walk Centrality and the Deformed Graph Laplacian. In section 3 we gave a simple example where the NBTW centrality in Definition 3.2 could be computed from first principles. To obtain a general-purpose algorithm, we quote two results from the theory of zeta functions of graphs that concern the combinatorics of NBTWs. Although originally derived from a pure mathematics viewpoint, these results turn out to be extremely useful from the perspective of matrix computations in network science, and they also highlight a connection between NBTWs and the deformed graph Laplacian. Lemma 5.1 gives a recurrence relation between NBTW counts of different lengths. Theorem 5.2 is an immediate corollary that gives an expression for the associated generating function.

Lemma 5.1. [13, 43, 44] Recall that $\Delta$ denotes the diagonal degree matrix and $p_{r}(A)$ has $(i, j)$ element that counts the number of NBTWs of length $r$ from $i$ to $j$. Then $p_{1}(A)=A, p_{2}(A)=A^{2}-\Delta$, and for $r>2$

$$
A p_{r-1}(A)=p_{r}(A)+(\Delta-I) p_{r-2}(A) .
$$

Theorem 5.2. [13, 43, 44] Let $\Phi(A, t):=\sum_{r=0}^{\infty} p_{r}(A) t^{r}$, where, for convenience, we set $p_{0}(A)=I$, and recall that $M(t)$ denotes the deformed graph Laplacian associated with $A$. Suppose moreover that $t$ is such that the power series converges. Then,

$$
M(t) \Phi(A, t)=\left(1-t^{2}\right) I
$$

In Definition 3.2 we see that the NBTW centrality $x_{i}$ of node $i$ may be computed via $\mathbf{x}=\Phi(A, t) \mathbf{1}$. From Theorem 5.2 we see that this simplifies to the linear system

$$
M(t) \mathbf{x}=\left(1-t^{2}\right) \mathbf{1}
$$

We note from (4.1) that, for any fixed value of $t, M(t)$ in (5.3) has the same sparsity structure as the coefficient matrix $I-\alpha A$ that appears in the original Katz system (2.2). Hence, NBTW centrality may be computed at the same cost as Katz centrality.

6. Further Spectral Analysis of the Deformed Graph Laplacian. Theorem 6.1 is an enhancement of Theorem 4.7 that relies on the results of Section 5 .

THEOREM 6.1. Suppose, with the notation and assumptions of Theorem 4.7, that $\lambda \in \mathbb{C}$ is a finite eigenvalue of both $M(t)$ and $\widetilde{M}(t)$. Then, the algebraic multiplicities of $\lambda$ as an eigenvalue of $M(t)$ and $\widetilde{M}(t)$ are the same.

Proof. We show that if we remove from the graph of $A$ just one leaf then the algebraic multiplicity of the finite eigenvalue $\lambda$ is preserved. The statement on algebraic multiplicities will then easily follow by induction on the number of leaves. Without loss of generality, let the leaf to be removed be node 1 and its unique adjacent node 
be node 2, and denote by $\widehat{A}$ (resp. $\widehat{M}(t)$ ) the adjacency matrix (resp. deformed graph Laplacian) of the graph obtained by removing node 1 and edge $1 \leftrightarrow 2$. Manifestly,

$$
(1 \oplus \widehat{M}(t))=M(t)-\left[\begin{array}{cccc}
0 & -t & 0 & \ldots \\
-t & t^{2} & 0 & \ldots \\
0 & 0 & 0 & \ldots \\
\vdots & \vdots & \vdots & \ddots
\end{array}\right]=M(t)-\left[\begin{array}{cc}
0 & -t \\
-t & t^{2} \\
0 & 0 \\
\vdots & \vdots
\end{array}\right]\left[\begin{array}{cccc}
1 & 0 & 0 & \ldots \\
0 & 1 & 0 & \ldots
\end{array}\right] .
$$

By the matrix determinant lemma, and since $\operatorname{det}(1 \oplus \widehat{M}(t))=\operatorname{det} \widehat{M}(t)$, we have

$$
\operatorname{det} \widehat{M}(t)=\operatorname{det} M(t) \operatorname{det}\left(I_{2}-\left[\begin{array}{cccc}
1 & 0 & 0 & \ldots \\
0 & 1 & 0 & \ldots
\end{array}\right] M(t)^{-1}\left[\begin{array}{cc}
0 & -t \\
-t & t^{2} \\
0 & 0 \\
\vdots & \vdots
\end{array}\right]\right) \text {, }
$$

and hence

$$
\frac{\operatorname{det} \widehat{M}(t)}{\operatorname{det} M(t)}=\operatorname{det}\left(I_{2}-N(t)\left[\begin{array}{cc}
0 & -t \\
-t & t^{2}
\end{array}\right]\right)
$$

having denoted by $N(t)$ the top-left $2 \times 2$ block of $M(t)^{-1}$. We now exploit Theorem 5.2 and express $[N(t)]_{22}=(1+f(t)) /\left(1-t^{2}\right)$, with $f(t):=\sum_{r=1}^{\infty} a_{r} t^{r}$, where $a_{r}$ is the number of NBTWs of length $r$ from node 2 to itself. Since node 1 is a leaf, and again by Theorem 5.2, we observe that

$$
[N(t)]_{11}=\frac{1+t^{2} f(t)}{1-t^{2}}, \quad[N(t)]_{12}=N(t)_{21}=\frac{t+t f(t)}{1-t^{2}} .
$$

By direct computation, $\operatorname{det}\left(I-N(t)\left[\begin{array}{cc}0 & -t \\ -t & t^{2}\end{array}\right]\right)=1$, so $\operatorname{det} \widehat{M}(t)=\operatorname{det} M(t)$.

We now work towards Theorem 6.3, which characterizes the graphs whose deformed graph Laplacian has an eigenvalue of modulus $<1$. To this end, Lemma 6.2 explicitly lists all the finite eigenvalues of the deformed graph Laplacian of a connected graph having average degree 2 .

Lemma 6.2. Let $A \in \mathbb{R}^{n \times n}$ be the adjacency matrix of a simple, undirected, connected graph whose average degree is precisely 2 , and let $M(t)$ be the associated deformed graph Laplacian. Then, $M(t)$ has precisely $\nu$ distinct finite eigenvalues, equal to the $\nu$ th complex roots of unity: $\lambda_{k}=\exp (2 k \pi i / \nu)$, for $0 \leq k \leq \nu-1$, where $3 \leq \nu \leq n$ is the length of the unique cycle in the graph of $A$. Moreover, the algebraic multiplicity of $\lambda_{k}$ is always 2 , whereas its geometric multiplicity is 2 if $\lambda_{k} \neq \pm 1$ or it is 1 otherwise.

Proof. Note first that, since the average degree is 2, the graph of $A$ can be obtained by adding one extra edge to a tree (see as an illustration Figure 6.1). This remark guarantees that there is a unique cycle in the graph of $A$. Furthermore, the resulting graph is bipartite if and only if $\nu$ is even. It follows by Proposition 4.4 that 1 is always an eigenvalue of $M(t)$ whereas -1 is an eigenvalue if and only if $\nu$ is even.

By Theorems 4.7 and 6.1, and by iteratively removing the leaves of the graph of $A$, we see that it suffices to prove the statement for the case when the graph of $A$ is a cycle of length $\nu$. 


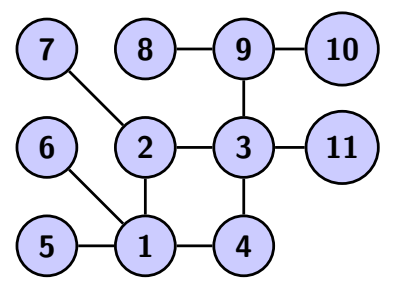

Fig. 6.1. A connected graph with average degree 2. This graph has 11 nodes, 11 edges, and one cycle of length 4. A tree can be obtained by removing any one of the four edges of the unique cycle, i.e., $1 \leftrightarrow 2,2 \leftrightarrow 3,3 \leftrightarrow 4$ or $4 \leftrightarrow 1$. This graph is bipartite as the cycle has even length: if we replaced, for example, $3 \leftrightarrow 4$ by $4 \leftrightarrow 11$, the unique cycle would have length 5 and the graph would no longer be bipartite.

In this case, as noted already in [36], the deformed graph Laplacian is (permutation similar to) a circulant matrix polynomial of the form

$$
M(t)=\left[\begin{array}{ccccc}
1+t^{2} & -t & & & -t \\
-t & 1+t^{2} & -t & & \\
& \ddots & \ddots & \ddots & \\
& & -t & 1+t^{2} & -t \\
-t & & & -t & 1+t^{2}
\end{array}\right] \in \mathbb{R}[t]^{\nu \times \nu} .
$$

By standard results in the theory of circulant matrices (or by induction on $\nu$ ), it is readily seen that $\operatorname{det} M(t)=\left(t^{\nu}-1\right)^{2}$. This shows that the $\nu$ th roots of unity are all eigenvalues with algebraic multiplicity 2 .

It remains to prove the statement on the geometric multiplicity. For the eigenvalues 1 and (if $\nu$ is odd) -1 , the statement follows by items 1-2 in Proposition 4.6. Otherwise, let $\lambda \neq \pm 1$ satisfy $\lambda^{\nu}=1$. Then the columns of the matrix

$$
N(\lambda)=\left[\begin{array}{cc}
\lambda^{\nu-1} & 1 \\
\lambda^{\nu-2} & \lambda \\
\vdots & \vdots \\
\lambda & \lambda^{\nu-2} \\
1 & \lambda^{\nu-1}
\end{array}\right]
$$

are linearly independent. Indeed, the determinant of the $2 \times 2$ leading submatrix of $N(\lambda)$ is $\lambda^{-2}\left(\lambda^{2}-1\right) \neq 0$. It is immediate to check that $M(\lambda) N(\lambda)=\mathbf{0}$, which concludes the proof. $\square$

TheOrem 6.3. Let $A \in \mathbb{R}^{n \times n}$ be the adjacency matrix of a simple undirected graph, and let $M(t)$ be the associated deformed graph Laplacian. Then, there exists a finite eigenvalue $\lambda$, with $|\lambda|<1$, of $M(t)$ if and only if the graph of $A$ has at least one connected component whose average degree is $>2$.

Proof. By Proposition 4.5, we can assume without loss of generality that the graph of $A$ is connected.

Suppose first that the average degree is $<2$. Then, the graph of $A$ is a tree, and the statement follows from Theorem 4.7 or, more directly, from Corollary 7.1. Similarly, if the average degree is precisely 2 , then the statement follows by Lemma 6.2.

To conclude the proof, suppose that the average degree of the graph of $A$ is $>2$. Let $f(t)$ denote $\operatorname{det} M(t)$ for $0 \leq t \leq 1$. We will argue that $f(0)=1, f(1)=0$, 
and $f^{\prime}(1)>0$, implying by elementary analysis the existence of $\lambda \in(0,1)$ such that $f(\lambda)=0$.

That $f(0)=1$ and $f(1)=0$ is an immediate consequence of $M(0)=I$ and $M(1)=\Delta-A=: L$. We have $d=\sum_{i} \operatorname{deg}_{i}>2 n$. Moreover,

$$
f^{\prime}(t)=\frac{\partial \operatorname{det} M(t)}{\partial t}=\operatorname{trace}\left(\operatorname{adj} M(t) \cdot M^{\prime}(t)\right)
$$

where $\operatorname{adj} X$ denotes the matrix adjugate of $X$. Evaluating at $t=1$,

$$
f^{\prime}(1)=\operatorname{trace}(\operatorname{adj} L \cdot(2 \Delta-A-2 I))=-2 \operatorname{trace}(\operatorname{adj} L)+\operatorname{trace}(\operatorname{adj} L \cdot A),
$$

where for the last equality we exploited the relation $\operatorname{adj} L \cdot L=0$. Let $\lambda_{1} \geq \cdots \geq$ $\lambda_{n-1} \geq \lambda_{n}=0$ be the eigenvalues of $L$ and set $p=\prod_{i=1}^{n-1} \lambda_{i}$. It is known [35] that $p>0$ if the graph of $A$ is connected. It is straightforward to show that

$$
\operatorname{adj} L=\frac{p}{n} \mathbf{1 1}^{T}
$$

implying $\operatorname{trace}(\operatorname{adj} L)=p$ and $\operatorname{trace}(\operatorname{adj} L \cdot A)=p d / n$. Hence, $f^{\prime}(1)=p\left(\frac{d}{n}-2\right)>0 . \mathbf{\square}$

We now have a detailed picture of the finite spectrum of $M(t)$ in terms of the features of the underlying graph. By Proposition 4.5 and Theorems 4.7 and 6.1, we may focus on the case of a simple connected graph with no leaves. Table 6.1 then summarizes our results on the finite spectrum and includes an additional result that we state below as Proposition 7.5.

TABLE 6.1

Spectra of the deformed graph Laplacian $M(t)$ for various simple, undirected, connected graphs. Note that the condition of being bipartite is equivalent to not having any cycles of odd length. The symbol? refers to properties not studied in this paper.

\begin{tabular}{c|c|c|c|c} 
Graph & E'value(s) & Alg. mult. & Geom. mult. & Comments \\
\hline Tree & 1 & 1 & 1 & always \\
& -1 & 1 & 1 & always \\
\hline Cycle of & 1 & 2 & 1 & always \\
length $\nu$ & -1 & 2 & 1 & iff bipartite \\
& $\lambda: \lambda^{\nu}=1, \lambda \neq \pm 1$ & 2 & 2 & always \\
\hline Graph with & 1 & 1 & 1 & always \\
more than & -1 & 1 & 1 & iff bipartite \\
one cycle & $\mu \in \mathbb{R}: \rho(A)^{-1}<\mu<1$ & 1 & 1 & always \\
& $\lambda \in \mathbb{C}: \mu<|\lambda| \leq 1$ & $?$ & $?$ &
\end{tabular}

7. The Radius of Convergence of the Generating Function. The power series $\sum_{r=0}^{\infty} p_{r}(A) t^{r}$ makes sense mathematically for any $t \in \mathbb{C}$; although, as mentioned in section 3 , for network analysis it is natural to focus on $t \in(0,1) \subset \mathbb{R}$. In this subsection we study the radius of convergence of this power series to its generating function $\left(1-t^{2}\right) M(t)^{-1}$. We note that it may happen that 1 or -1 are within the radius of convergence but are also eigenvalues of $M(t)$, so that the latter is not invertible at $t=1$ or $t=-1$. In this case, and with slight abuse of notation, when talking of $\left(1-t^{2}\right) M(t)^{-1}$ for $t=1$ or $t=-1$ we tacitly mean the appropriate limit of this expression for $t \rightarrow 1$ or $t \rightarrow-1$. (The existence of the limit in this scenario follows from the analysis below and standard results on rational functions). 
First, we note that, by construction, elementwise it holds that

$$
p_{k}(A)=\left|p_{k}(A)\right| \leq\left|A^{k}\right|=A^{k} .
$$

Hence, $|t|<\rho(A)^{-1}$, where $\rho(A)$ is the spectral radius of $A$, surely suffices for convergence, since $\sum_{k=0}^{\infty} A^{k} t^{k}$ has radius of convergence $\rho(A)^{-1}$.

However, this condition is sufficient but not necessary, as shown by the star graph example of section 3 , where $\rho(A)=\sqrt{n-1}$ yet we have convergence for all $t$. More generally, if the graph $A$ is a tree (or a forest) so that there are no cycles, then $p_{k}(A)=0$ for large enough values of $k$. It follows that $\Phi(A, t)$ is a polynomial in $t$, and the series converges for all $t$. This implies that $M(t)$ cannot have any finite eigenvalues other than \pm 1 ; and both 1 and -1 must be eigenvalues by Proposition 4.4 (and noting that any tree is bipartite). Since the average degree of any tree is $<2$, by Proposition 4.6 these eigenvalues are semisimple. This observation yields the following corollary on the spectral properties of $M(t)$ in the case of a forest.

Corollary 7.1. Suppose that $A$ is the adjacency matrix of a forest, and let $M(t)$ be the associated deformed graph Laplacian. Then, $M(t)$ has the only finite eigenvalues 1 and -1. Moreover, 1 and -1 are both semisimple eigenvalues. (By Proposition 4.2, these two properties are equivalent to $\ell_{n}(t)=t^{2}-1$, where $\ell_{n}(t)$ is the nth invariant polynomial of $M(t)$, as defined in Theorem 4.1.)

For a general $A$, our analysis is based on the properties of the deformed graph Laplacian $M(t)$ as a matrix polynomial. The following technical lemma will be useful. It follows from the conditional converse to Abel's Theorem on power series [16].

Lemma 7.2. For any $z \in \mathbb{C}$ let $\sum_{k=0}^{\infty} a_{k} z^{k}$ be a power series with nonnegative real coefficients, i.e., $a_{k} \geq 0 \forall k \in \mathbb{N}$. Suppose that the power series converges to the rational function $p(z) / q(z)$, with $p(z), q(z) \in \mathbb{R}[z]$ coprime polynomials, with radius of convergence $r>0$. Then, $q(r)=0$.

We are now ready to state our main result on the convergence of the matrix power series $\sum_{r} p_{r}(A) t^{r}$. It turns out that it is determined by a particular eigenvalue of $M(t)$.

THEOREM 7.3. Let $A$ be the adjacency matrix of a simple, undirected, graph. Let $M(t)=I-t A+t^{2}(\Delta-I)$ be the associated deformed graph Laplacian, and let $\ell_{n}(t)$ be the nth invariant polynomial of $M(t)$, as defined in Theorem 4.1.

The radius of convergence of the power series in Theorem 5.2 is equal to $|\chi|$ where $\chi$ is the smallest (in modulus) zero of

$$
r(t):=\frac{\ell_{n}(t)}{1-t^{2}}
$$

while $\chi:=\infty$ if such a function does not vanish.

Moreover, let $\lambda$ be the smallest (in modulus) eigenvalue of the matrix polynomial $M(t)$.

1. There exists $\mu \in(0,1] \subset \mathbb{R}$ such that (i) $\mu=|\lambda|$ and (ii) $\mu$ is an eigenvalue of $M(t)$.

2. If $\mu<1$, then $|\chi|=\mu$.

3. If $\mu=1$ is a semisimple eigenvalue of $M(t)$, then the underlying graph is a forest and $\chi=\infty$.

4. If $\mu=1$ is a defective eigenvalue of $M(t)$, then $|\chi|=\mu=1$.

Before proving Theorem 7.3, we give a few remarks that should be kept in mind.

- By Proposition 4.2, being a zero of $\ell_{n}(t)$ is equivalent to being a finite eigenvalue of $M(t)$, and being a semisimple eigenvalue of $M(t)$ is equivalent to being a simple zero of $\ell_{n}(t)$. 
- By Proposition 4.6, if the underlying graph does not have any connected component with average degree 2 , then 1 is a semisimple eigenvalue of $M(t)$, otherwise it is defective.

- By Proposition 4.2, any zero of $r(t)$ is a finite eigenvalue of $M(t)$.

- Again by Proposition 4.2, if $M(t)$ has at least one finite eigenvalue $\neq \pm 1$, or if at least one among 1 or -1 is a defective eigenvalue of $M(t)$, then $r(t)$ must have at least one zero.

- If -1 is an eigenvalue of $M(t)$, which happens for example if the graph of $A$ is bipartite, then $r(t)$ is actually a polynomial.

- The power series converges for any $t \in \mathbb{C}$ if and only if $r(t)$ does not vanish for any $t \in \mathbb{C}$, if and only if $\ell_{n}(t)=1-t^{2}$.

Proof. [of Theorem 7.3] From Theorem 5.2, for any $t \in \mathbb{C}$ within the disk of convergence, the matrix power series converges to $(1-t)^{2} M(t)^{-1}$. Since $M(t)$ is polynomial, $\Phi(A, t)=\left(1-t^{2}\right) M(t)^{-1}$ is a matrix rational function in $t$ (but generally not another matrix polynomial). Observe that

$$
M(t)^{-1}=\frac{\operatorname{adj} M(t)}{\operatorname{det} M(t)},
$$

where $\operatorname{adj} X$ denotes the matrix adjugate of $X$. The entries of $\operatorname{adj} M(t)$ are, up to a sign, the $(n-1) \times(n-1)$ minors of $M(t)$. Let $g_{n-1}(t)$ be the monic GCD of the entries of $\operatorname{adj} M(t)$, and $g_{n}(t)=\kappa \operatorname{det} M(t)$ where $\kappa \neq 0$ is such that $g_{n}(t)$ is monic. Then, we see that $t_{0} \in \mathbb{C}$ is a pole of $\left(1-t^{2}\right) M(t)^{-1}$ if and only if it is a zero of

$$
\frac{g_{n}(t)}{g_{n-1}(t)\left(1-t^{2}\right)}=\frac{\ell_{n}(t)}{1-t^{2}}
$$

where we used Theorem 4.1. Hence, the radius of convergence must be equal to $|\chi|$, where $\chi$ is the smallest (in modulus) zero of the rational function $r(t)$ in (7.1) if any, or $\chi=\infty$ if $r(t)$ does not have any zero. We may now address the remaining four points in the theorem.

1. Note that $\ell_{n}(t) \in \mathbb{R}[t]$ and that by construction $p_{k}(A) \geq 0$ elementwise. If the radius of convergence is $\leq 1$, then the existence of a positive real eigenvalue $\mu$ such that $\mu=|\lambda|$ follows by Proposition 4.2 and by applying Lemma 7.2 elementwise. On the other hand, if the radius of convergence is $>1$, then clearly $\mu=1$, because 1 is an eigenvalue by Proposition 4.4 and because by Proposition 4.2 there cannot be roots of $r(t)$ of modulus $\leq 1$. Finally, $\mu=|\lambda|>0$ follows by Proposition 4.4.

2. If $\mu<1$, then $r(\mu)=0$ but $r(t) \neq 0$ for $|t|<\mu$, so it follows by the argument above that $\chi=\mu$.

3. Suppose $|\chi|<1$, then $\ell_{n}(\chi)=0$, and hence $\operatorname{det} M(\chi)=0$. Hence $\chi$ is an eigenvalue of $M(t)$ of modulus strictly less than 1 , thus contradicting $\mu=$ $|\lambda|=1$. Moreover, by Theorem 4.8, if $|\chi| \geq 1$ then either $|\chi|=1$ or $\chi=\infty$. On the other hand, by Proposition 4.6, since 1 is a semisimple eigenvalue there is no connected component of the underlying graph whose average degree is precisely 2 ; and by Theorem 6.3 we can exclude the possibility of a connected component with average degree $>2$, since otherwise we would have $\mu=|\lambda|<$ 1. Hence, the graph is a forest and therefore $\chi=\infty$ by Corollary 7.1.

4. If $\mu=1$ is a defective eigenvalue of $M(t)$, then by Proposition 4.6 it is a multiple root of $\ell_{n}(t)$, and hence $r(1)=0$. On the other hand, for any $|t|<1$, $\operatorname{det} M(t) \neq 0 \Rightarrow \ell_{n}(t) \neq 0 \Rightarrow r(t) \neq 0$. Thus, the radius of convergence is 1 . 
We note that, as a consequence of Theorem 7.3 and the fact that $|t|<\rho(A)^{-1}$ is a sufficient condition for convergence of the power series $\sum_{k} p_{k}(A) t^{k}$, the following corollary gives a lower bound for $\mu$ which is tighter than zero.

COROLlaRY 7.4. Let $M(t)$ be the deformed graph Laplacian associated with a graph with adjacency matrix $A$ whose spectral radius is $\rho(A)$. Then, every eigenvalue $\lambda$ of $M(t)$ satisfies $|\lambda| \geq \rho(A)^{-1}$.

Theorem 7.3 reduces to the following simpler form assuming that $\mu<1$, which by Theorem 6.3 is equivalent to assuming that the underlying graph has at least one connected component with average degree $>2$. We note that this result therefore covers a general case that is likely to be encountered frequently in practice.

Proposition 7.5. Suppose that the matrix polynomial $M(t)=I-t A+t^{2}(\Delta-I)$ has an eigenvalue $\lambda$ with $|\lambda|<1$. Then there exists a positive real number $\mu$ such that $\mu$ is the smallest (in modulus) eigenvalue of $M(t)$. Moreover, the radius of convergence of the power series $\sum_{k=0}^{\infty} t^{k} p_{k}(A)$ is equal to $\mu$.

Furthermore, if the graph of $A$ is connected, $\mu$ is a simple eigenvalue of $M(t)$ and every other finite eigenvalue $\lambda$ satisfies $\mu<|\lambda| \leq 1$.

Proof. Except for the last sentence, the statement is an immediate corollary of Theorem 7.3.

To prove the last sentence, observe that by Theorem 6.3 the graph of $A$ must have average degree $>2$, and hence, it must have at least two cycles. Moreover, by Theorem 6.1 we may assume that the graph of $A$ has no leaves. Consider the directed graph obtained by replacing each undirected edge by two directed edges, with opposite orientations, between the same nodes; we label these directed edges $1, \ldots, 2 m$. By Corollary 1 and equation (2.3) in [26], $\lambda \neq \pm 1$ is a finite eigenvalue of $M(t)$ if and only if $\lambda^{-1} \neq 0, \pm 1$ is an eigenvalue of the $2 m \times 2 m$ matrix $B$ defined as follows: if there exists a NBTW on the dircted graph that includes the consecutive directed edges $i$ and $j$ then $B_{i j}=1$, otherwise $B_{i j}=0 . B$ is a nonnegative matrix. Suppose it is reducible, then there exist two directed edges $i$ and $j$ such that there is no NBTW of the form $\cdots i \cdots j \cdots$. This contradicts the assumption that the original undirected graph is connected, without leaves, and with at least two cycles.

Hence, $B$ is nonnegative and irreducible, and the statement follows by the PerronFrobenius theorem.

8. Practical Observations. In practice, given a specific, real network, we would like to know what range of choices are available for the parameter $t$ in (5.3). From Proposition 7.5, in the generic case where there is at least one connected component with average degree $>2$, the strict upper limit for $t$ is given by $\lambda$, the smallest (in modulus) eigenvalue of $M(t)$. From the theory of matrix polynomials $[24,32,34,37,38]$, one can show that this $\lambda$ may be equivalently defined as the reciprocal of the largest (in modulus) eigenvalue of the matrix

$$
C:=\left[\begin{array}{cc}
A & I-\Delta \\
I & 0
\end{array}\right]
$$

This matrix is known as the companion linearization [24] of rev $M(t)$, the reversal matrix polynomial of $M(t)$ [34, 38]; note that it also appears in [33, Equation (17)]. Since only the largest eigenvalue of a very sparse matrix is needed, it is feasible to compute $\lambda$ for many large networks. Should this computation turn out to be impossible or unreliable, other approaches to give a lower bound on $|\lambda|$ are available, and we give a brief overview below. 
For example, one could use the generalized Gershgorin theorem [5, Theorem 3.1], obtaining that, if $\operatorname{deg}_{j}$ is the $j$ th diagonal element of the matrix $\Delta$, then no finite eigenvalue of $M(t)$ can possibly lie in the region

$$
\bigcap_{j=1}^{n}\left\{\lambda \in \mathbb{C}:\left|1+\left(\operatorname{deg}_{j}-1\right) \lambda^{2}\right|>\operatorname{deg}_{j}|\lambda|\right\}
$$

which, denoting by $\operatorname{deg}_{\max }$ the maximum degree of the graph of $A$, for $|\lambda|<1$ can be shown to reduce to $\left\{\left|1+\left(\operatorname{deg}_{\max }-1\right) \lambda^{2}\right|>\operatorname{deg}_{\max }|\lambda|\right\}$.

Alternatively, bounds specific for matrix polynomials based on tropical algebra exist $[6,39]$. For example, by $[39$, Theorem 3.1 , item(ii)] if $\|\cdot\|$ is any induced matrix norm then $|\lambda| \geq r$ where $r$ is the unique positive root of the quadratic polynomial $x^{2}\|\Delta-I\|+x\|A\|-1$. Solving the quadratic equation gives

$$
|\lambda| \geq \frac{\sqrt{\|A\|^{2}+4\|\Delta-I\|}-\|A\|}{2\|\Delta-I\|} .
$$

Now, $\|A\|_{2}=\rho(A)$ while $\|A\|_{1}=\|A\|_{\infty}=\operatorname{deg}_{\max }$, whereas (assuming that the graph of $A$ has at least one edge, so that $\Delta \neq 0)\|\Delta-I\|=\operatorname{deg}_{\max }-1$ for all the three norms considered above. Since $\rho(A)<\operatorname{deg}_{\max }$, the spectral norm gives the strongest of the three bounds:

$$
|\lambda| \geq \frac{\sqrt{\rho(A)^{2}+4 \operatorname{deg}_{\max }-4}-\rho(A)}{2 \operatorname{deg}_{\max }-2}
$$

and we deduce that a sufficient condition for convergence is

$$
0<t<\frac{\sqrt{\rho(A)^{2}+4 \operatorname{deg}_{\max }-4}-\rho(A)}{2 \mathrm{deg}_{\max }-2} .
$$

Theorem 4.7 shows that the spectrum of $M(t)$ does not change when a leaf is removed from the graph. It follows that, as a practical approach, we can preprocess the graph by iteratively removing all leaves. Suppose this gives a matrix $\hat{A}$ of dimension $\widehat{n} \times \widehat{n}$. Then the techniques in this section for computing or bounding the radius of convergence may be applied to $\widehat{A}$, rather than $A$, leading to a gain in efficiency when $\widehat{n} \ll n$, that is, when there are many, or large, trees dangling off the graph. Furthermore, the linear system (5.3) for NBTW centrality may be solved with $\widehat{A}$. The centrality $x_{L}$ for any node $L$ that was removed as a leaf is then recoverable via the iterative relation $x_{L}=1+t\left(x_{N}-t\right)$. Here, $x_{N}$ denotes the centrality of the node that was the neighbour of node $L$ at the stage where node $L$ was removed ${ }^{3}$.

We also mention that recursive leaf removal corresponds to finding the 2-core of a graph. It is interesting to note that computing $k$-cores, and corresponding $k$-shells, has itself been proposed as a way to identify influential spreaders in a network [29]; although recent work has pointed out limitations of this approach [41].

9. Connection with M-matrix Theory. Suppose that $0 \leq t<\min \{1,|\chi|\}$ where $\chi$ is defined as in Theorem 7.3. Theorem 7.3 implies that the power series

\footnotetext{
${ }^{3}$ By contrast, leaf pruning reduces $\rho(A)$ in general, and hence cannot be used in this way to gain efficiency in the choice of $\alpha$ for standard Katz centrality. However, this type of dimension reduction can reduce the cost of solving the resulting Katz and PageRank systems; see [2, 31].
} 
$\sum_{r} p_{r}(A) t^{r}$ converges to $\left(1-t^{2}\right) M(t)^{-1}$, and as a consequence $M(t)^{-1} \geq 0$ elementwise. In this section, we give a more direct proof of this fact using the theory of M-matrices. The proof relies on the observation that $M(t)$ is a Hermitian matrix polynomial, and employs arguments that are typical in the theory of Hermitian matrix polynomials [34].

By Theorem 7.3, the two conditions $t<|\chi|$ and $t<1$ imply that $t$ is strictly smaller than the smallest real positive eigenvalue of $M(t)$. Now, let $M$ be the matrix obtained by evaluating $M(t)$ at $t=t_{0}$ for some $0 \leq t_{0}<|\chi|$. Note that $M(0)=I$ has $n$ positive eigenvalues. Since the eigenvalues of a matrix depending continuously on a parameter $t$ are continuous as a set (see [27, Ch. II], [34, Sec. 5] for more details), and since $M(t)$ is symmetric and not singular for all $t \in[0, \mu)$, necessarily $M=M\left(t_{0}\right)$ has all positive eigenvalues as well.

Now, note that $M$ is a Z-matrix [40], i.e., its off-diagonal elements are $\leq 0$. By [40, Theorem 1], a Z-matrix such that all its eigenvalues have positive real part is an M-matrix. Hence, $M$ is an M-matrix. As a consequence, again by [40, Theorem 1], $M^{-1}$ is a nonnegative matrix.

10. Limiting Behavior of the NBTW centrality Measure. In $[4$, Theorem 5.1], Benzi and Klymko study the limiting behaviour of classical parameter-dependent centrality measures for undirected networks, letting the parameter tend to the endpoints of the convergence interval. In this section we study the limiting behaviour of the NBTW centrality measure.

THEOREM 10.1. Let $A$ be the adjacency matrix of a simple, connected, undirected graph, let $M(t)$ be the associated deformed graph Laplacian, and let $\mathbf{x}(t)$ be the vector of the corresponding $N B T W$ centrality measure defined as in Definition 3.2 or equivalently in (5.3), with $t \in(0,|\chi|)$ and $\chi$ defined as in Theorem 7.3.

1. As $t \rightarrow 0^{+}$, the rankings produced by $\mathbf{x}(t)$ converge to those produced by the vector of the degree centralities.

2. Suppose further that $|\chi| \leq 1$, so that by Theorem 7.3 we have $0<|\chi|=\mu \leq 1$, where $\mu$ is the smallest positive eigenvalue of $M(t)$. Then as $t \rightarrow|\chi|^{-}$the rankings produced by $\mathbf{x}(t)$ converge to those produced by the eigenvector $\mathbf{v}$ of the matrix polynomial $M(t)$ associated with the eigenvalue $\mu$.

Proof. Part 1 may be proved by essentially the same argument given in $[4$, Proof of Theorem 5.1, item (i)], except that one has to replace $[f(t A) \mathbf{1}]_{i}$ by $\left[\left(1-t^{2}\right) M(t)^{-1} \mathbf{1}\right]_{i}$. Indeed, expanding in powers of $t$,

$$
\left[\left(1-t^{2}\right) M(t)^{-1} \mathbf{1}\right]_{i}=\left[I \mathbf{1}+A t \mathbf{1}+p_{2}(A) t^{2} \mathbf{1}+\ldots\right]_{i},
$$

which for $t \rightarrow 0^{+}$behaves as $1+\operatorname{deg}_{i} t+O\left(t^{2}\right)$, where $\operatorname{deg}_{i}$ is the degree of the $i$ th node of the graph of $A$. Hence, the statement follows.

For part 2, observe first that $\mu$ has geometric multiplicity 1 as an eigenvalue of $M(t)$. This follows from Proposition 7.5 if $\mu<1$ and from Proposition 4.4 if $\mu=1$. Note that, for any fixed $t<\mu, M(t)$ is a symmetric matrix which can be orthogonally diagonalized, say, $M(t)=Q(t) \Lambda(t) Q(t)^{T}$, and hence

$$
\mathbf{x}(t)=\left(1-t^{2}\right) Q(t) \Lambda(t)^{-1} Q(t)^{T} \mathbf{1} .
$$

Moreover, $Q(t)$ and $\Lambda(t)$ can be taken to be real-analytic functions of $t[27,34]$. Now, let $\mathbf{v}$ be the eigenvector of the matrix polynomial $M(t)$ associated with the eigenvalue $\mu$, and $\|\mathbf{v}\|_{2}=1$. Then, the matrix $M(\mu)$ has a simple eigenvalue 0 and $M(\mu) \mathbf{v}=\mathbf{0}$. Let $\lambda_{1}(t), \lambda_{2}(t), \ldots, \lambda_{n}(t)$ denote the eigenvalues of the matrix $M(t)$ for 
any fixed $0<t<\mu$, where the labelling is such that $\lambda_{1}(\mu) \geq \lambda_{2}(\mu) \geq \cdots \geq \lambda_{n}(\mu)$. Letting $\mathbf{q}_{i}(t)$ be the corresponding normalized eigenvectors, we can expand (10.1) as $\mathbf{x}(t)=\left(1-t^{2}\right) \mathbf{q}_{n}(t)^{T} \mathbf{1} \lambda_{n}^{-1}(t) \mathbf{q}_{n}(t)+O\left(\lambda_{n}(t) / \lambda_{n-1}(t)\right)$. Now, observing that ${ }^{4}$

$$
\hat{\mathbf{x}}(t)=\frac{\lambda_{n}(t)}{\left(1-t^{2}\right) \mathbf{q}_{n}^{T}(t) \mathbf{1}} \mathbf{x}(t)
$$

gives the same rankings as $\mathbf{x}(t)$ for any $0<t<\mu$, since the two vectors are proportional. Noting that $\mathbf{q}_{n}(t) \rightarrow \mathbf{v}$ and $\lambda_{n}(t) / \lambda_{n-1}(t) \rightarrow 0$ as $t \rightarrow \mu^{-}$, we have that $\hat{\mathbf{x}}(t) \rightarrow \mathbf{v}$ for $t \rightarrow \mu^{-}$, yielding the statement.

Theorem 10.1 has the following, somewhat surprising, consequence: a PerronFrobenius-like result for the deformed graph Laplacian.

THEOREM 10.2. Let $A$ be the adjacency matrix of a simple, connected, undirected graph and $M(t)$ be the corresponding deformed graph Laplacian. Let $\mu \leq 1$ be the smallest real positive eigenvalue $\mu$ of $M(t)$. Then, the corresponding eigenvector $\mathbf{v}$ can be chosen to be componentwise nonnegative.

Equivalently, under the same assumptions, the eigenvector associated with the dominant eigenvalue $\mu^{-1}$ of the matrix $C$ in (8.1) can be chosen to be componentwise nonnegative.

Proof. The first part of the statement is an immediate consequence of Theorem 10.1. The second part follows from the first part and the theory of companion linearizations of monic matrix polynomials, see for example [24, 32, 37, 38].

Note that, by [33, Equation (17)], the limiting behaviour of the NBTW centrality measure characterized in Theorems 10.1 and 10.2 corresponds precisely to the nonbacktracking eigenvector centrality introduced in [33]. It is worth emphasizing that this is generally different from the classical eigenvector centrality, thus showing that (unlike classical centrality measures) our newly proposed centrality measure does not follow the "universal" limiting behaviour for large $t$ described in [4, Theorem 5.1].

At this stage it is useful to return to the star example (3.1). We know from the first principles derivation in section 3, or from Corollary 7.1 and Theorem 7.3, that the radius of convergence for the generating function is infinite. In this case the nonbacktracking eigenvector centrality in [33], which can be computed via "the leading eigenvector" of the matrix $C$ in (8.1), corresponds to the limit $t \rightarrow 1$. This measure has the attraction of being parameter free. However, in this example there are two dominant eigenvalues at \pm 1 . In order to obtain nonnegative entries in the corresponding eigenvector, we must choose the eigenvalue +1 . The associated eigenvector corresponds to the null space of the graph Laplacian, and hence it is the vector of all ones. So, nonbacktracking eigenvector centrality assigns the same value to all nodes in the star. We regard this as unsatisfactory behavior, caused by the aim of completely eliminating the localization effect. Indeed, ranking the center of a star above its neighbors has been suggested as a basic "axiom" for centrality measures, $[7,22]$.

The more general NBTW centrality measure introduced in this work may then be regarded as a means to interpolate between this extreme case and the opposite extreme of degree centrality.

\footnotetext{
${ }^{4}$ Note that here we require $\mathbf{q}_{n}^{T}(t) \mathbf{1} \neq 0$. This is true because, by our previous analysis, evaluating $M^{-1}(t)$ for any fixed value of $t \in(0, \mu)$ we obtain a nonnegative matrix (see also section 9). Hence, by the Perron-Frobenius Theorem, the eigenvector corresponding to the smallest eigenvalue of the matrix $M(t)$, i.e., $\mathbf{q}_{n}(t)$, has all nonnegative components, implying $\mathbf{q}_{n}^{T}(t) \mathbf{1}>0$.
} 
11. Synthetic Example: Galaxy Network. We now present a class of large scale networks where constraining to NBTWs can be shown to give qualitatively different, and potentially beneficial, results. We consider the case where $m$ copies of the star graph with adjacency matrix (3.1) are joined together via a single central node which is linked to the central node of each star. Figure 11.1 illustrates the case where $m=3$ and $n=9$. We will refer to such a structure as a galaxy graph.

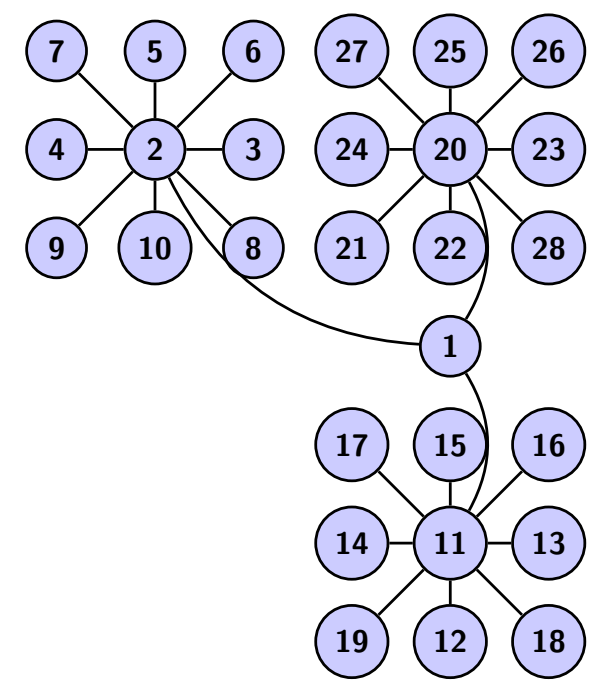

FIG. 11.1. A galaxy graph with $m=3$ stars, each with $n=9$ vertices. Having labelled the vertices as within the figure, for this galaxy graph we say that the vertex 1 is galaxy-central, the vertices 2,11,20 are star-central, and every other vertex is peripheral.

We wish to analyze the walk-based centralities in detail. In each case, by symmetry, there are at most three distinct values for the entries of the centrality vector $\mathbf{x}$, corresponding to the three types of node. Along with $x_{1}$, we will take $x_{2}$ as a representative of the star-central nodes (with indices $x_{k n+2}$ for $k=0,1, \ldots, m-1$ ) and $x_{3}$ as a representative for the remaining peripheral nodes.

For degree centrality (which is also the $\alpha \rightarrow 0^{+}$and $t \rightarrow 0^{+}$limit of the other centralities considered) we clearly have $x_{1}=m, x_{2}=n$ and $x_{3}=1$. Hence, for $m>n$, the galaxy-central node ranks highest. In the case $m \leq n$, there is still a case to be made for node 1 to be the most central - intuitively it is best positioned to initiate traversals around the network. We show below that even with $m=O(1)$ as $n \rightarrow \infty$, it is possible for NBTW centrality to rank the galaxy-central node highest, whereas Katz and eigenvalue centrality always place $x_{2}>x_{1}$.

Still labelling by $A$ the adjacency matrix of the star graph, and letting $\mathbf{e}_{1}=$ $\left[\begin{array}{llll}1 & 0 & \ldots & 0\end{array}\right]^{T} \in \mathbb{R}^{n}$, the galaxy graph has adjacency matrix

$$
B=\left[\begin{array}{cccc}
0 & \mathbf{e}_{1}^{T} & \ldots & \mathbf{e}_{1}^{T} \\
\mathbf{e}_{1} & A & & \\
\vdots & & \ddots & \\
\mathbf{e}_{1} & & & A
\end{array}\right] .
$$

For Katz centrality, it is useful to know the eigenvalues of $B$. Define $\mathbf{a}_{ \pm}=(n-$ $1)^{-1 / 4}\left[\begin{array}{llll} \pm \sqrt{(n-1) / 2} & \sqrt{1 / 2} & \ldots & \sqrt{1 / 2}\end{array}\right]^{T} \in \mathbb{R}^{n}$. Then $A=\mathbf{a}_{+} \mathbf{a}_{+}{ }^{T}-\mathbf{a}_{-} \mathbf{a}_{-}{ }^{T}$. Let 
$Q$ be any orthogonal matrix whose first two columns are $\mathbf{a}_{+} /\left|\mathbf{a}_{+}\right|$and $\mathbf{a}_{-} /\left|\mathbf{a}_{-}\right|$, then $Q^{T} A Q=D:=\operatorname{diag}(\sqrt{n-1},-\sqrt{n-1}, 0, \ldots, 0)$. Moreover, by $Q\left(\mathbf{e}_{1}-\mathbf{e}_{2}\right)=\sqrt{2} \mathbf{e}_{1}$ we deduce that $Q^{T} \mathbf{e}_{1}=2^{-1 / 2}\left(\mathbf{e}_{1}-\mathbf{e}_{2}\right)=: \mathbf{v}$ and hence $B$ is similar to

$$
\left[\begin{array}{cccc}
0 & \mathbf{v}^{T} & \ldots & \mathbf{v}^{T} \\
\mathbf{v} & D & & \\
\vdots & & \ddots & \\
\mathbf{v} & & & D
\end{array}\right],
$$

which in turn is permutation similar to (denoting by $\mathbf{1} \in \mathbb{R}^{m}$ the vector of all ones)

$$
0_{m(n-2)} \oplus\left[\begin{array}{ccc}
0 & \mathbf{1}^{T} / \sqrt{2} & -\mathbf{1}^{T} / \sqrt{2} \\
\mathbf{1} / \sqrt{2} & \sqrt{n-1} \cdot I_{m} & 0 \\
-\mathbf{1} / \sqrt{2} & 0 & -\sqrt{n-1} \cdot I_{m}
\end{array}\right] .
$$

Computing the characteristic polynomial of the $(2 m+1) \times(2 m+1)$ matrix above is immediate. For example, Schur complementing we get

$$
\left(\lambda^{2}-(n-1)\right)^{m}\left(\lambda-\frac{m}{2} \frac{2 \lambda}{\lambda^{2}-(n-1)}\right)=\lambda\left(\lambda^{2}-(n-1)\right)^{m-1}\left(\lambda^{2}-(m+n-1)\right) .
$$

We conclude that the Katz measure requires $0<\alpha<(m+n-1)^{-1 / 2}$.

The Katz system, $(I-\alpha B) \mathbf{x}=\mathbf{1}$, solves directly to give

$$
x_{1}=\frac{1+(m-1)(n-1) \alpha^{2}+m \alpha}{1-(m+n-1) \alpha^{2}}, \quad x_{2}=\frac{1+n \alpha}{1-(m+n-1) \alpha^{2}},
$$

and

$$
x_{3}=\frac{1+\alpha+\alpha^{2}(1-m)}{1-(m+n-1) \alpha^{2}} .
$$

For NBTW centrality, we may work from first principles.

- The galaxy-central node 1 has $m$ NBTWs of length one, $m(n-1)$ NBTWs of length two, and no NBTW of length $\geq 3$.

- From each star-central node, such as node 2, there are $n$ NBTWs of length one, $m-1$ NBTWs of length two, $(m-1)(n-1)$ NBTWs of length three, and no NBTW of length $\geq 4$.

- From each peripheral node, such as node 3 , begin one NBTW of length one, $n-1$ NBTWs of length two, $m-1$ NBTWs of length three, $(m-1)(n-1)$ NBTWs of length four, and no NBTWs of length $\geq 5$.

Hence, we have

$$
x_{1}=1+m t+m(n-1) t^{2}, \quad x_{2}=1+n t+(m-1) t^{2}+(m-1)(n-1) t^{3},
$$

and

$$
x_{3}=1+t+(n-1) t^{2}+(m-1) t^{3}+(m-1)(n-1) t^{4} .
$$

Whether $x_{1}>x_{2}$ depends, for either centrality index, not only on the parameters, $\alpha$ or $t$, but also on $m$ and $n$.

For both Katz and NBTW centrality, imposing $x_{1}>x_{2}$ yields that $\alpha$ (resp. $t$ ) must be greater than the critical value $(n-m) /((m-1)(n-1))$. If $m \geq n$, both 
centrality measures give $x_{1}>x_{2}$ for all valid parameter values - the degree centrality ranking prevails. However, if $n>m$, it could happen that $1 / \rho(B)$ is smaller than this critical value, and hence Katz fails to have a transition to $x_{2}>x_{1}$ when NBTW does.

Katz fails to have such a transition when

$$
(n-m)^{2}(m+n-1)>(n-1)^{2}(m-1)^{2}
$$

and one way to achieve this when $n \rightarrow \infty$ is if $m=O\left(n^{\gamma}\right)$ with $\gamma \leq 1 / 2$.

For example, let us analyze what happens if $m=O(1)$ while letting $n$ grow. We see in (11.1) that Katz always gives $x_{2}>x_{1}$ in this regime, but for the NBTW version in (11.2), the choice of $t$ is crucial. To be concrete, fixing $m=5$ we find that the inequality (11.4) is satisfied for $n>21$. Recall that $\alpha<1 / \sqrt{n+4}$ in this setting. We obtain for the Katz centrality

$$
\frac{x_{1}}{x_{2}}=\frac{1+4(n-1) \alpha^{2}+5 \alpha}{1+n \alpha}
$$

which implies that $x_{1} / x_{2}=O(1 / \sqrt{n})$ when $n$ grows and $\alpha$ is a given fraction of its upper bound. Conversely, for the NBTW centrality

$$
\frac{x_{1}}{x_{2}}=\frac{1+5 t+5(n-1) t^{2}}{1+n t+4 t^{2}+4(n-1) t^{3}} .
$$

Here, $x_{1}>x_{2}$ for $t>(n-5)(4 n-4) \approx O(1 / 4)$, and, for example, setting $t=1 / 2$ yields $x_{1} / x_{2} \approx 5 / 4$ for large $n$.

For further comparison, we note that since we know that the largest eigenvalue of $B$ is $\sqrt{m+n-1}$, and again using the symmetry, it is straightforward to compute the Perron-Frobenius eigenvector of $B$, and hence, the eigenvector centrality for the galaxy graph. We find that

$$
\frac{x_{1}}{x_{3}}=m, \quad \frac{x_{2}}{x_{3}}=\sqrt{m+n-1} \Longrightarrow \frac{x_{1}}{x_{2}}=\frac{m}{\sqrt{m+n-1}} .
$$

Again, we note that for $n>m^{2}$ this yields $x_{2}>x_{1}$.

In summary, in this example restricting to nonbacktacking walks can produce dramatically different results, and can highlight the galaxy-central node even when its degree is arbitrarily smaller than that of the star-central nodes.

We also recall the behavior of nonbacktracking eigenvector centrality on a star graph, as discussed at the end of section 10. The same effect arises here: taking the $t \rightarrow 1^{-}$limit in (11.2) and (11.3), we see that all nodes are assigned the same centrality value by this measure.

\section{Tests on Real Data.}

12.1. Localization. We begin this section with a test on a small network where the results may be visualized easily. Figure 12.1 concerns a 40 node network with 42 edges. The network is based on a sample from the Barabási and Albert preferential attachment model [3] generated by calling pref $(40,1)$ in the CONTEST toolbox for MATLAB [45]. To avoid having a tree, we also added three extra edges, creating three cycles. The model produces "scale free" networks where many nodes have low degree and a small proportion have high degree. In this case the degrees present in the network are 17 (one node), 7 (one node), 5 (one node), 3 (two nodes), 2 
TABLE 12.1

Twitter networks for ten UK cities. First and second row: original number of nodes and percentage of nodes that remain after pruning. Third and fourth row: same information for edges. Fifth and sixth row: spectral radius $\rho(A)$ relevant for Katz and $\rho(C)$ relevant for $N B T W$. Seventh row: Kendall's $\tau$ correlation between Katz and NBTW centrality for the top 100 Katz-ranked nodes.

\begin{tabular}{ccccccccccc} 
& Edinb. & Glasg. & Card. & Brist. & Nott. & Birm. & Sheff. & Leeds & Manch. & Lond. \\
\hline Nodes & 1645 & 1802 & 2685 & 2892 & 2066 & 1321 & 2845 & 5263 & 7646 & 16171 \\
Remain & $41 \%$ & $36 \%$ & $45 \%$ & $46 \%$ & $44 \%$ & $41 \%$ & $44 \%$ & $46 \%$ & $44 \%$ & $42 \%$ \\
\hline Edges & 2146 & 2284 & 4444 & 4538 & 3155 & 1993 & 4399 & 9319 & 12163 & 24266 \\
Remain & $55 \%$ & $49 \%$ & $67 \%$ & $65 \%$ & $63 \%$ & $61 \%$ & $64 \%$ & $70 \%$ & $65 \%$ & $61 \%$ \\
\hline$\rho(A)$ & 7.8 & 8.0 & 20.3 & 14.0 & 13.9 & 12.0 & 15.4 & 30.8 & 20.0 & 22.3 \\
$\rho(C)$ & 5.5 & 5.1 & 18.8 & 12.1 & 12.3 & 10.3 & 13.5 & 28.5 & 15.6 & 20.7 \\
Corr. & 0.80 & 0.65 & 0.95 & 0.87 & 0.86 & 0.85 & 0.92 & 0.98 & 0.83 & 0.96
\end{tabular}

(fourteen nodes) and 1 (twenty-one nodes). The adjacency matrix $A$ and the twoby-two block matrix $C$ in (8.1) have spectral radius $\rho(A)=4.26$ and $\rho(C)=1.39$. For the Katz and NBTW centrality measures, we used $90 \%$ of the upper limit; that is, $\alpha=0.9 / \rho(A)=0.21$ for Katz and $t=0.9 / \rho(C)=0.65$ for NBTW. Figure 12.1 displays the network twice, with the size of each node proportional to the centrality, using Katz on the left and NBTW on the right. We see that the NBTW version is less closely tied to the nodal degree, and in particular does not emphasize the high degree node to the same extent. The lower picture in Figure 12.1 scatter plots the two centrality measures, normalized to have maximum component equal to one. This further clarifies that NBTW centrality has delocalized the high degree node, and also shows that the two measures give different rankings, even at the high endthe top 10 nodes, in descending order, are 1,2,13,4,8,7,21,3,12,6 for Katz and $1,2,7,13,21,17,36,4,8,3$ for NBTW.

Looking at the localization effect in larger networks, with $n=50,000$ we called pref $(n, 2)$ and added an extra 100 undirected edges uniformly at random, deleting repeated edges. Using 500 independent preferential attachment network samples of this type, the average inverse participation ratio (3.8) for degree centrality was found to be 0.06 , and for Katz with $\alpha=0.9 / \rho(A)$ it was the same order of magnitude at 0.05 . For NBTW centrality with $t=0.9 / \rho(C)$, this value decreased by an order of magnitude to 0.003. (In all cases the standard error was below the precision displayed.)

12.2. Pruning. Next, we illustrate the effect of iteratively pruning the leaves from a network, as discussed in section 8. On the left in Figure 12.2 we show the largest connected component of a protein-protein interaction network for yeast [46]. There are 564 nodes and 687 edges. After removing leaves until none remain, we arrive at the network on the right, with 167 nodes and 290 edges. Using larger protein-protein interaction networks from the Integrated Interactions Database [30] (downloaded on 13th of June, 2016), we found that leaf pruning reduced the number of nodes and edges, respectively, as follows; worm: $4853 \mapsto 2628,12635 \mapsto 10480$; fly: $9139 \mapsto 7303,49959 \mapsto 48146$; mouse: $7569 \mapsto 4583,19268 \mapsto 16346$. Similarly, Table 12.1 summarizes results for reciprocated mention Twitter networks in ten UK cities, taken from [25]. We see that more than half of the nodes and typically around a third of the edges are eliminated. We conclude that pruning to reduce problem size is a viable option for some real problem classes.

12.3. Further Comparisons. In rows five and six of Table 12.1 we show, for each city, $\rho(A)$ for the orginal adjacency matrix and $\rho(C)$ for the block matrix in (8.1). The final row of the table shows the correlation between the Katz and NBTW centrality measures. More precisely, we used $\alpha=0.9 / \rho(A)$ for Katz and $t=0.9 / \rho(C)$ 
Katz

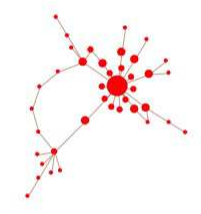

NBTW
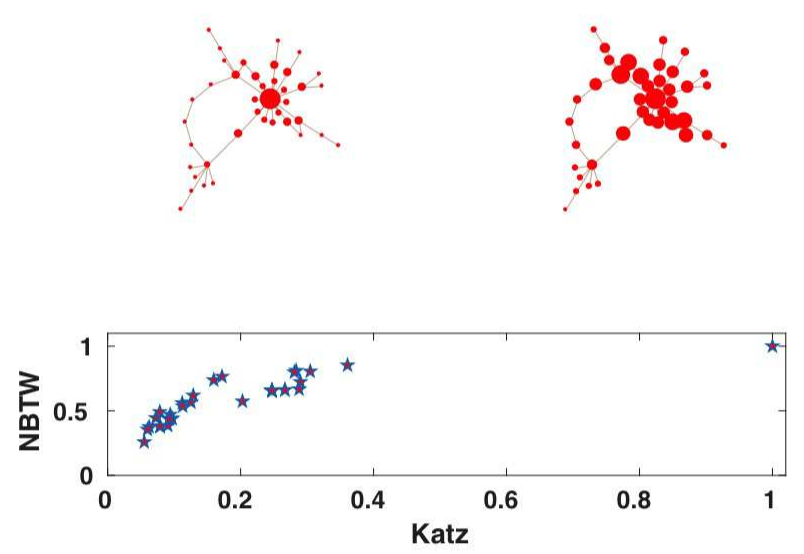

FIG. 12.1. Centrality measures for a preferential attachment network. Upper pictures have node size proportional to Katz (left) and NBTW (right) centrality. Lower picture scatter plots the two centrality measures.

for NBTW. We then found the top 100 nodes according to the Katz measure, and for these nodes, computed the correlation between the two centrality measures. Following the recommendations in [42], we quantify correlation with Kendall's $\tau$ coefficient. The spectral radii are seen to be close and the correlations high, with the noteworthy exception of Glasgow.

To give more details on the Glasgow data, Figure 12.3 shows the underlying network and Figure 12.4 scatter plots the two centrality measures for the top 100 Katz nodes. In both pictures, we have highlighted three nodes of interest. The square marks a node of degree 44 that is top-ranked by both measures. This node has the second highest degree in the network. The node with highest degree, 45, is marked with a star. This node is ranked second by Katz, but only sixth by NBTW. The triangle marks another node where the measures noticeably disagree. This node, of degree 8 , is ranked ninth by Katz and fifth by NBTW. Figure 12.5 zooms in to the network in order to show some local detail. The highest degree (star) node has 15 

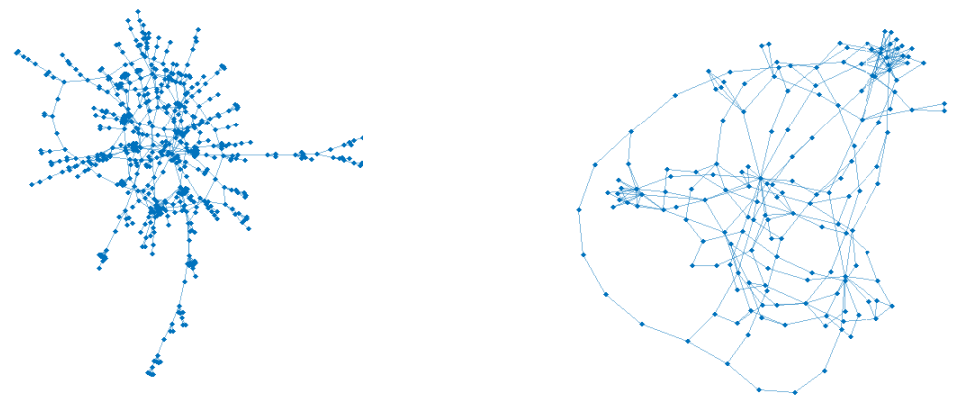

FIG. 12.2. A yeast protein-protein interaction network. Left: original network. Right: remaining network after leaves have been iteratively pruned until none remain.

leaves among its 45 neighbors, and hence can initiate many short backtracking walks. The second highest degree (square) node has only 4 leaves among its 44 neighbors. The lower degree (triangle) node has 8 neighbors, none of which are leaves. Taking the top 10 nodes in each ranking, from first to tenth, and recording their degrees, we obtain 44, 45, 26, 24, 21, 13, 25, 11, 8, 10 for Katz and 44, 26, 24, 13, 8, 45, 10, 11, 10, 5 for NBTW. Overall, this example shows how the new NBTW measure (i) is able to move further from basic degree centrality through the use of a larger downweighting parameter, and (ii) gives less credence to low quality, peripheral neighbors.

We finish by using a network where some external information is available concerning importance. We use a yeast protein interaction network that has been studied in $[17,18,21]$. There are $n=2224$ nodes connected by 6609 edges. Each node represents a protein, and in addition to the interaction data there is an independently produced list indicating which proteins are essential; for these proteins, the cell dies if the corresponding gene is made inoperative. There are 589 essential proteins, which is around $26 \%$ of the total.

In Figure 12.6 we give a visualization of the network, with essential proteins marked by a darker circle. The spectral radius of the underlying adjacency matrix $A$ is $\rho(A)=19.0$; whereas the spectral radius of $C$ is $\rho(C)=17.3$.

In Figure 12.7, we write $\operatorname{Katz}(0.5)$ to describe the standard Katz centrality vector in (2.2) with downweighting parameter $\alpha=0.5 / \rho(A)$. Similarly Katz(0.75) describes the case where $\alpha$ is $0.75 / \rho(A)$. The figure also gives results for eigenvector centrality, which corresponds to the limit $\alpha \rightarrow 1 / \rho(A)$ from below. We compare these three measures with degree centrality (which may be viewed as $\operatorname{Katz}(0)$ ). For each plot in Figure 12.7, we consider all integers $1 \leq k \leq 100$ and compute the difference between (a) how many of the top $k$ nodes ranked by that centrality measure are essential and (b) how many of the top $k$ nodes ranked by degree centrality are essential. We see that increasing $\alpha$ generally improves the performance in terms of including essential proteins among the top-ranked nodes - the height of the plots typically increases with $\alpha$. We note that the underlying degree centrality measure identifies 43 of the essential proteins in its top 100.

Figure 12.8 shows the nonbacktracking analogue of Figure 12.7. More precisely, we write $\operatorname{NBTW}(0.5)$ and $\operatorname{NBTW}(0.75)$ to describe the NBTW centrality vector in (5.3) 


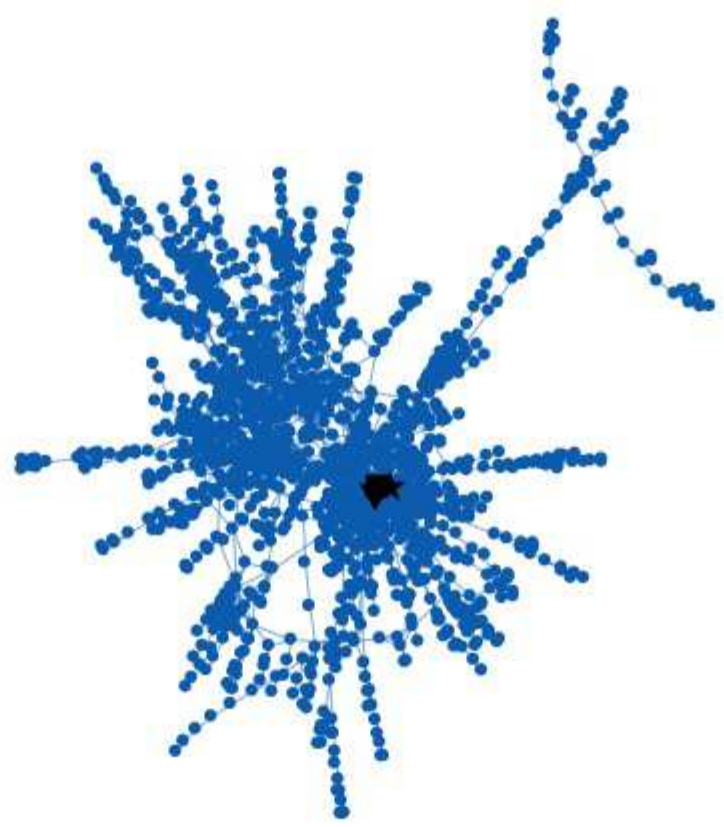

FIG. 12.3. Visualization of the Glasgow network from Table 12.1. Three interesting nodes are marked with a black triangle, star and square. (See Figure 12.5 for a zoom.)

with downweighting parameter $t=0.5 / \rho(C)$ and $t=0.75 / \rho(C)$, respectively. We also test the nonbacktraking eigenvector centrality measure of [33], which, according to Theorems 10.1 and 10.2 and the subsequent discussion, corresponds to the limit $t \rightarrow$ $1 / \rho(C)$ from below. We see similar behavior to that in Figure 12.7, where increasing the attenuation parameter leads to better identification of essential proteins.

In Figure 12.9, we show the difference between the number of essential proteins in the top $k$ lists for NBTW eigenvector and standard eigenvector centrality. We see that the difference is typically zero or positive; further investigation showed that the same essential proteins are being indentified in each case, with the nonbacktracking version generally ranking them slightly higher in the list.

13. Summary. Our aim in this work was to motivate, define and analyse a new walk-based network centrality measure. Our practical take-home message is that backtracking walks can be eliminated at no extra cost, and, moreover, in doing so we have a greater range of freedom in our choice of Katz-style downweighting parameter. We proved that this parameter allows us to interpolate between standard degree centrality and the recently proposed nonbacktracking eigenvector centrality of 


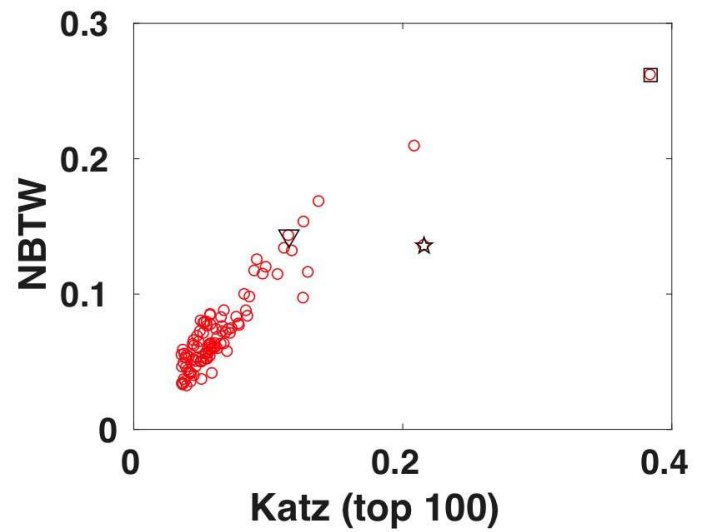

FIG. 12.4. Glasgow network from Figure 12.3: Kendall's $\tau$ correlation between Katz and NBTW centrality for the 100 top-ranked Katz nodes. Three interesting nodes are marked with a black triangle, star and square.

[33], which was specially designed to eliminate localization effects but, based on our rigorous analysis of star-like graphs, may be regarded as draconian.

Our work exploited results from the theory of zeta functions of graphs in order to open up a new and fruitful connection between network science and matrix polynomial theory. In this way we have posed and answered a range of novel and practically important questions concerning the deformed graph Laplacian. From the matrix polynomial perspective, we (i) characterized the spectrum of the deformed graph Laplacian in terms of features of the underlying graph (see Table 6.1), (ii) showed that the convergence of the associated power series is governed by the smallest deformed graph Laplacian eigenvalue (Theorem 7.3 and Proposition 7.5) and (iii) established a Perron-Frobenius type result (Theorem 10.2). From a practical perspective, in section 8 , we derived computable bounds on the choice of downweighting parameter. Further, we showed that since the deformed graph Laplacian spectrum is invariant under the removal of leaves (Theorem 4.7), the idea of restricting attention to nonbacktracking walks has the potential to give computational gains for tree-like networks.

Acknowledgements We thank Kate Fenu, Chris Godsil, Federico Poloni and Francesco Tudisco for useful discussions on this work. We also thank Noël MalodDognin and Nataša Pržulj for providing us with data downloaded from [30], and Ernesto Estrada for supplying the data used in Figures 12.6 to 12.9. Finally, we are grateful to two anonymous referees for their comments which helped us to improve the presentation.

\section{REFERENCES}

[1] N. Alon, I. Benjamini, E. Lubetzky, and S. Sodin, Non-backtracking random walks mix faster, Communications in Contemporary Mathematics, 09 (2007), pp. 585-603. 


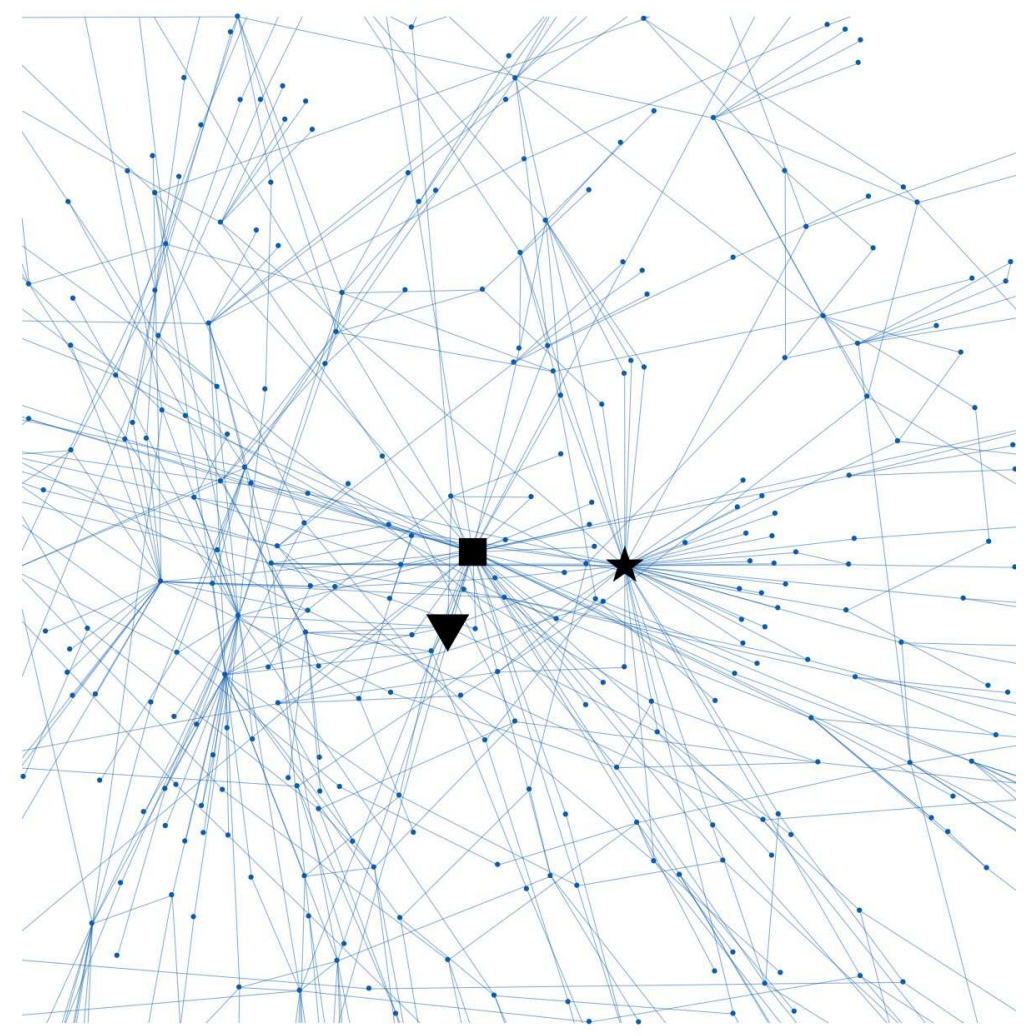

FIG. 12.5. Zoom of the visualization in Figure 12.3.

[2] A. ArRatia And C. MarijuÁn, On graph combinatorics to improve eigenvector-based measures of centrality in directed networks, Linear Algebra and Its Applications, 504 (2016), pp. 325353.

[3] A. L. Barabási And R. Albert, Emergence of scaling in random networks, Science, 286 (1999), pp. 509-512.

[4] M. Benzi AND C. KLYMKo, On the limiting behavior of parameter-dependent network centrality measures, SIAM J. Matrix Anal. Appl., 36 (2015), pp. 686-706.

[5] D. BINdel AND A. Hood, Localization theorems for nonlinear eigenvalues, SIAM J. Matrix Anal. Appl., 34 (2013), pp. 1728-1749.

[6] D. A. Bini, V. Noferini, AND M. Sharify, Locating the eigenvalues of matrix polynomials, SIAM J. Matrix Anal. Appl., 34 (2013), pp. 1708-1727.

[7] P. Boldi And S. Vigna, Axioms for centrality, Internet Mathematics, 10 (2014), pp. 222-262.

[8] B. Bollobas, Modern Graph Theory, Springer, New York, NY, 1998.

[9] P. Bonacich, Factoring and weighting approaches to status scores and clique identification, Journal of Mathematical Sociology, 2 (1972), pp. 113-120.

[10] — Power and centrality: a family of measures, American Journal of Sociology, 92 (1987), pp. 1170-1182.

[11] S. P. Borgatti, Centrality and network flow, Social Networks, 27 (2005), pp. 55-71.

[12] S. P. Borgatti and M. G. Everett, A graph theoretic perspective on centrality, Social Networks, 28 (2006), pp. 466-484.

[13] R. Bowen AND O. E. LANFORd, Zeta functions of restrictions of the shift transformation, 
Essential Proteins

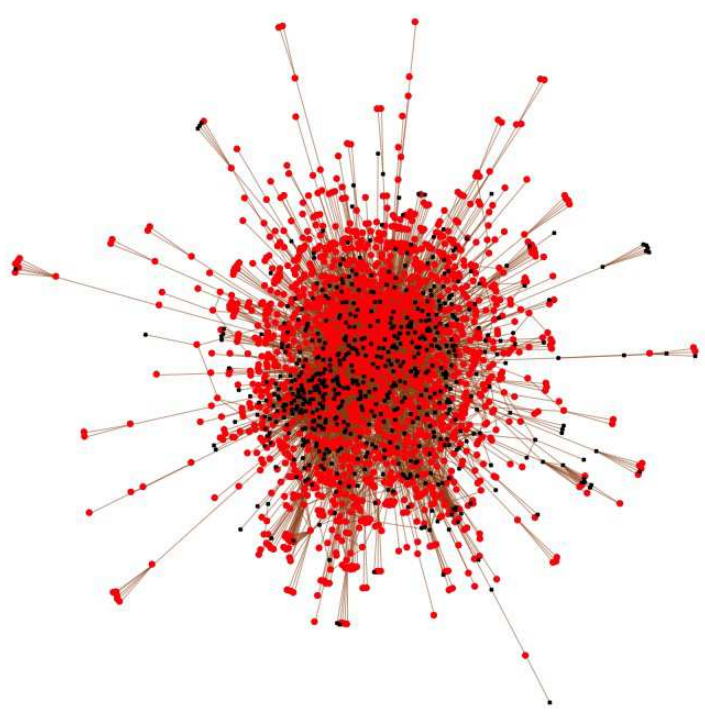

FIG. 12.6. The yeast protein interaction network from [17, 18, 21]. Essential nodes are marked with a darker symbol. (Color online.)

in Global Analysis: Proceedings of the Symposium in Pure Mathematics of the Americal Mathematical Society, University of California, Berkely, 1968, S.-S. Chern and S. Smale, eds., American Mathematical Society, 1970, pp. 43-49.

[14] D. Cvetkové, P. Rowlinson, And S. Simić, Eigenspaces of Graphs, Cambridge University Press, Cambridge, 1997.

[15] D. Cvetković, P. Rowlinson, And S. K. Simić, Signless Laplacians of finite graphs, Linear Algebra Appl., 423 (2007), pp. 155-171.

[16] H. Delange, The converse of Abel's Theorem on power series, Ann. of Math., 50 (1949), pp. 94-109.

[17] E. Estrada, Protein bipartivity and essentiality in the yeast protein-protein interaction network, Journal of Proteome Research, 5 (2006), pp. 2177-2184.

[18] - Virtual identification of essential proteins within the protein interaction network of yeast, Proteomics, 6 (2006), pp. 35-40.

[19] E. Estrada, The Structure of Complex Networks, Oxford University Press, Oxford, 2011.

[20] E. Estrada And D. J. Higham, Network properties revealed through matrix functions, SIAM Review, 52 (2010), pp. 696-671. 


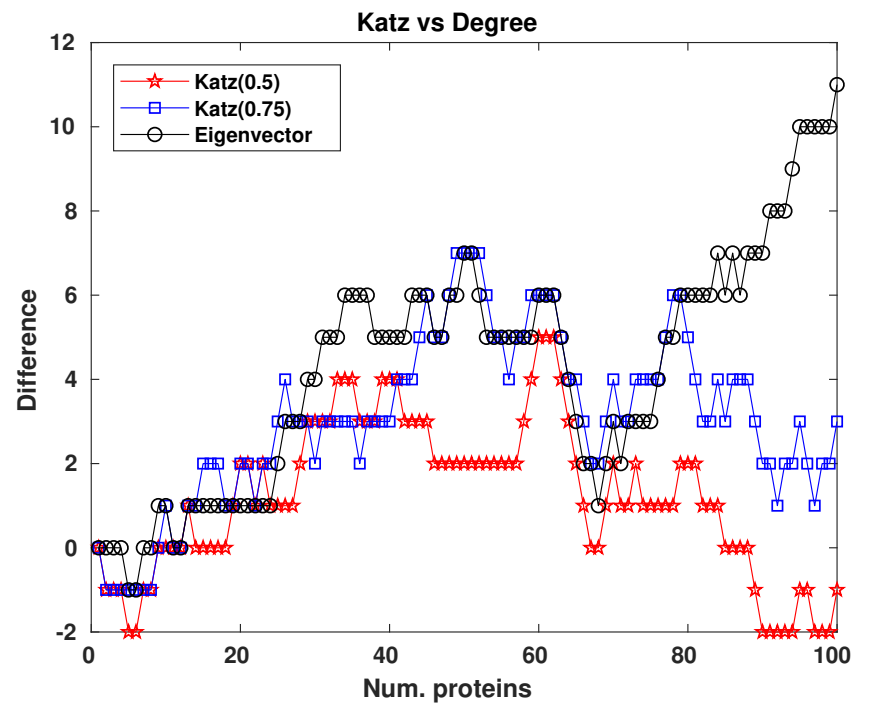

FIG. 12.7. The difference between the number of essential proteins in the top $k$ list for Katz centrality/eigenvector centrality and for degree centrality. Here Katz is computed with $\alpha=0.5 / \rho(A)$ and $\alpha=0.75 / \rho(A)$.

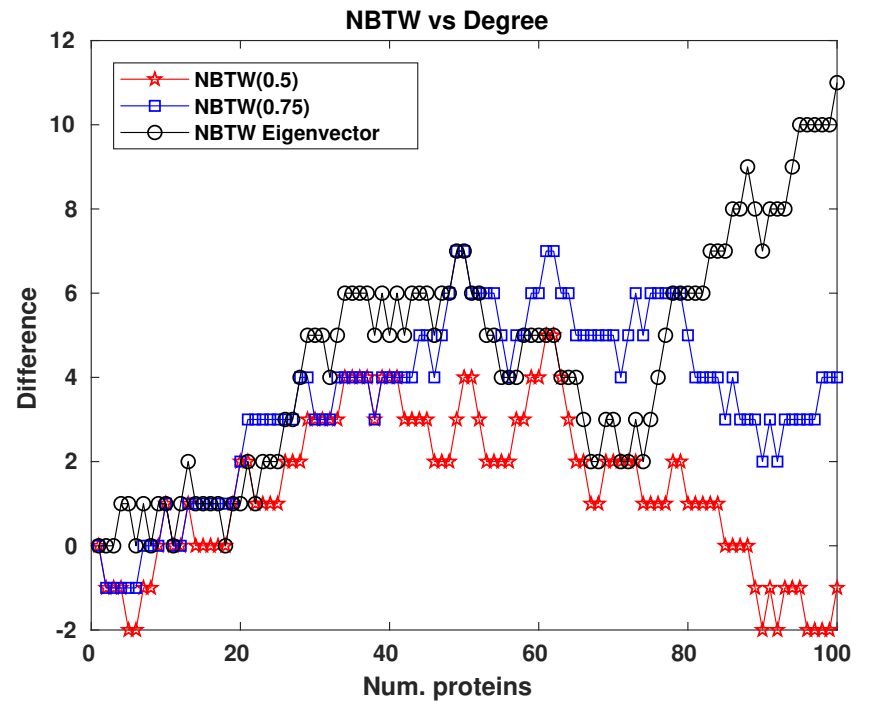

FIG. 12.8. The difference between the number of essential proteins in the top $k$ list for NBTW centrality/nonbacktracking eigenvector centrality and for degree centrality. Here NBTW centrality is computed with $t=0.5 / \rho(C)$ and $t=0.75 / \rho(C)$.

[21] E. Estrada And G. Silver, Accounting for the role of long walks on networks via a new matrix function, Journal of Mathematical Analysis and Applications, 449 (2017), pp. 1581-1600.

[22] L. C. Freeman, Centrality in social networks: conceptual clarification, Social Networks, 1 (1978), pp. 215-239.

[23] G. Frobenius, Theorie der linearen Formen mit ganzen Coefficienten, J. Reine Angew. Math. (Crelle), 86 (1878), pp. 146-208.

[24] I. Gohberg, P. Lancaster, and L. Rodman, Matrix Polynomials, SIAM, Philadelphia, PA, 2009. 


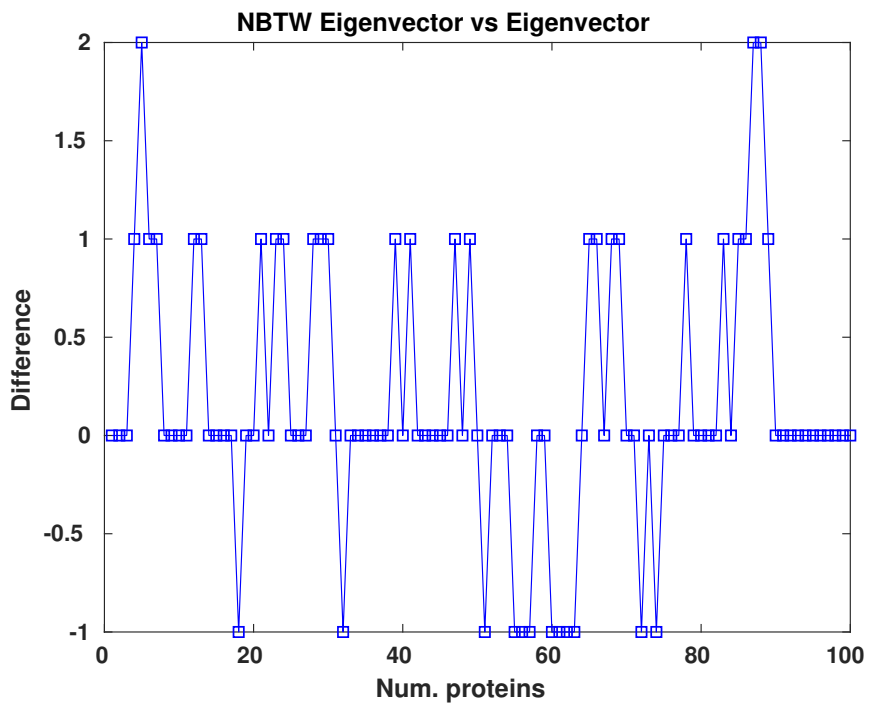

FIG. 12.9. The difference between the number of essential proteins in the top $k$ list for nonbacktracking eigenvector centrality and standard eigenvector centrality.

[25] P. Grindrod And T. E. LeE, Comparison of social structures within cities of very different sizes, Royal Society Open Science, 3 (2016).

[26] M. D. Horton, H. M. Stark, And A. A. Terras, What are zeta functions of graphs and what are they good for?, in Quantum graphs and their applications, G. Berkolaiko, R. Carlson, S. A. Fulling, and P. Kuchment, eds., vol. 415 of Contemp. Math., 2006, pp. 173-190.

[27] T. Kato, Perturbation Theory for Linear Operators, Springer-Verlag, New York, NY, 1966.

[28] L. KATZ, A new index derived from sociometric data analysis, Psychometrika, 18 (1953), pp. 39-43.

[29] M. Kitsak, L. K. Gallos, S. Havlin, F. Liljeros, L. Muchnik, H. E. Stanley, and H. A. MAKSE, Identification of influential spreaders in complex networks, Nature Physics, 6 (2010), p. 888.

[30] M. Kotlyar, C. Pastrello, N. Sheahan, and I. Jurisica, Integrated interactions database: tissue-specific view of the human and model organism interactomes, Nucleic Acids Research, 44 (2016), pp. 536-541.

[31] A. N. Langville and C. D. Meyer, Deeper inside PageRank, Internet Mathematics, 1 (2004), pp. $335-380$.

[32] D. S. Mackey, N. Mackey, C. Mehl, and V. Merhmann, Vector spaces of linearizations for matrix polynomials, SIAM J. Matrix Anal. Appl., 28 (2006), pp. 971-1004.

[33] T. Martin, X. Zhang, and M. E. J. Newman, Localization and centrality in networks, Phys. Rev. E, 90 (2014), p. 052808.

[34] V. Mehrmann, V. Noferini, F. Tisseur, and H. Xu, On the sign characteristics of Hermitian matrix polynomials, Linear Algebra Appl., 511 (2016), pp. 328-364.

[35] R. MerRis, Laplacian matrices of graphs: a survey, Linear Algebra Appl., 197-198 (1994), pp. $143-176$.

[36] F. Morbidi, The deformed consensus protocol, Automatica, 49 (2013), pp. 3049-3055.

[37] Y. Nakatsukasa, V. Noferini, and A. Townsend, Vector spaces of linearizations of matrix polynomials: a bivariate polynomial approach, SIAM J. Matrix Anal. Appl., (2016).

[38] V. Noferini And F. Poloni, Duality of matrix pencils, Wong chains and linearizations, Linear Algebra Appl., 471 (2015), pp. 730-767.

[39] V. Noferini, M. Sharify, AND F. Tisseur, Tropical roots as approximations to eigenvalues of matrix polynomials, SIAM J. Matrix Anal. Appl., 36 (2015), pp. 138-157.

[40] R. J. Plemmons, M-matrix characterizations. I-nonsingular M-matrices, Linear Algebra Appl., 18 (1977), pp. 175-188.

[41] F. RADicchi AND C. CASTEllano, Leveraging percolation theory to single out influential spreaders in networks, Phys. Rev. E, 93 (2016), p. 062314.

[42] D. Schoch, T. W. Valente, And U. Brandes, Correlations among centrality indices and a 
class of uniquely ranked graphs, Social Networks, 50 (2017), pp. 46-54.

[43] H. Stark and A. Terras, Zeta functions of finite graphs and coverings, Advances in Mathematics, 121 (1996), pp. 124-165.

[44] A. Tarfulea and R. Perlis, An Ihara formula for partially directed graphs, Linear Algebra and its Applications, 431 (2009), pp. 73-85.

[45] A. TAylor and D. J. Higham, CONTEST: A controllable test matrix toolbox for MATLAB, ACM Trans. Math. Softw., 35 (2009), pp. 26:1-26:17.

[46] P. Uetz, L. Giot, G. Cagney, T. A. Mansfield, R. S. Judson, J. R. Knight, E. LockShon, V. Narayan, M. Srinivasan, P. Pochart, A. Qureshi-Emili, Y. Li, B. Godwin, D. Conover, T. Kalbfleish, G. Vijayadamodar, M. Yang, M. Johnston, S. Fields, AND J. M. RothberG, A comprehensive analysis of protein-protein interactions in saccharomyces cerevisiae, Nature, 403 (2000), pp. 623-627.

[47] S. Wasserman and K. Faust, Social Network Analysis: Methods and Applications, Cambridge University Press, Cambridge, 1994. 